\title{
Resolving Toric Varieties with Nash Blowups
}

\author{
Atanas Atanasov, Christopher Lopez, Alexander Perry, Nicholas Proudfoot, and \\ Michael Thaddeus
}

\section{CONTENTS}

1. Introduction

2. Equivalence to a Combinatorial Problem

3. Resolution Trees

4. The 2-Dimensional Case

5. A Method for Enumerating Simplicial Cones

6. Results of Computer Investigations

Acknowledgments

References
2000 AMS Subject Classification: 14M25, 52B20

Keywords: toric varieties, Nash blowups, iteration, resolution of singularities
It is a long-standing question whether an arbitrary variety is desingularized by finitely many normalized Nash blowups. We consider this question in the case of a toric variety. We interpret the normalized Nash blowup in polyhedral terms, show how continued fractions can be used to give an affirmative answer for a toric surface, and report on a computer investigation in which over a thousand 3- and 4-dimensional toric varieties were successfully resolved.

\section{INTRODUCTION}

Let $X$ be a variety over an algebraically closed field $K$. Its Nash blowup is a variety over $K$ with a projective morphism to $X$, which is an isomorphism over the smooth locus. Roughly speaking, it parametrizes all limits of tangent planes to $X$ (a precise definition is given in Section 2 below). The Nash blowup of a singular $X$ is not always smooth but seems, in some sense, to be less singular than $X$. Strictly speaking, this is false, for in characteristic $p>0$, as explained in [Nobile 75], the plane curve $x^{p}-y^{q}=0$ is its own Nash blowup for any $q>0$. In this and other ways, the ordinary Nash blowup proves intractable.

However, let the normalized Nash blowup be the normalization of the Nash blowup. Then, of course, the normalized Nash blowup of every curve is smooth. The normalized Nash blowup of a surface can be singular, but as shown in [Hironaka 83] and [Gonzalez-Sprinberg 87, Spivakovsky 90], every surface becomes smooth after finitely many normalized Nash blowups. Thus we are drawn to ask the following question.

Question 1.1. Is every variety desingularized by finitely many normalized Nash blowups?

According to [Spivakovsky 90], Nash asked Hironaka this question in the early 1960s. An affirmative answer would give a canonical procedure for desingularizing an 
arbitrary variety. The answer to this question is not known and is surely difficult. In this paper we address a narrower question.

Question 1.2. Is every toric variety desingularized by finitely many normalized Nash blowups?

We do not answer this question conclusively either. But we do exhibit abundant evidence supporting an affirmative answer. Using the "toric dictionary," which translates every problem in toric geometry into a problem on convex polyhedra, we convert Question 1.2 into a problem amenable to computer calculation. Then we carry out this calculation for thousands of examples. In every case, finitely many Nash blowups produce a smooth toric variety.

\subsection{Summary of the Paper}

In Section 2 we introduce the toric dictionary and, following [Gonzalez-Sprinberg 77b], translate the question into convex geometry. In Section 3 we summarize the effect of the iterated Nash blowup in the toric case, using the notion of a resolution tree. In Section 4 we spell out what happens in the 2-dimensional toric case in terms of continued fractions.

In Section 5 we digress briefly on the classification of quasismooth affine toric varieties, those corresponding to simplicial cones in the toric dictionary. Then in Section 6 we give an account of our computer investigations.

\subsection{Notation and Conventions}

We slightly abuse terminology in two ways. First, since we are concerned with the normalized Nash blowup throughout, we refer to it simply as the Nash blowup. Second, since we are concerned with rational polyhedra and rational polyhedral cones throughout, we refer to them simply as polyhedra and cones. We denote the natural numbers, including 0 , by $\mathbb{Z}_{+}$, and we likewise denote the nonnegative rational numbers, including 0 , by $\mathbb{Q}_{+}$. We denote the span of $v_{1}, \ldots, v_{k}$ with coefficients in $S$ by $S\left\langle v_{1}, \ldots, v_{k}\right\rangle$. Thus, for example, the first quadrant in $\mathbb{Q}^{2}$ is denoted by $\mathbb{Q}_{+}\left\langle e_{1}, e_{2}\right\rangle$.

\section{EQUIVALENCE TO A COMBINATORIAL PROBLEM}

\subsection{Nash Blowups}

Let $X \subset \mathbb{P}^{n}$ be a quasiprojective variety of dimension $d$ over an algebraically closed field $K$. The Gauss map is the rational map $X \rightarrow \operatorname{Gr}(d+1, n+1)$ taking a smooth point to its tangent plane. The Nash blowup of $X$ is defined to be the closure of the graph of the Gauss map. The normalized Nash blowup of $X$ is the normalization of the Nash blowup of $X$. Gonzalez-Sprinberg's and Spivakovsky's results are concerned with this variant, as are ours. Consequently, we shall abuse terminology by referring to a normalized Nash blowup simply as a Nash blowup.

Remark 2.1. As defined, the Nash blowup appears to depend on the projective embedding of $X$, but it can be reformulated in terms of Kähler differentials and hence depends only on $X$ (and makes sense even if $X$ is not quasiprojective) [Gonzalez-Sprinberg 87].

Remark 2.2. Since the normalization of a variety over $K$ is a finite morphism [Hartshorne 77, I 3.9A] and the pullback of an ample bundle by a finite morphism is ample [Lazarsfeld 04, 1.7.7], the normalization is a projective morphism. Hence the natural morphism from the (normalized) Nash blowup of $X$ to $X$ is projective.

Remark 2.3. Clearly the Nash blowup of a smooth variety is itself, and the Nash blowup of a product is a product.

Remark 2.4. If $X \subset \mathbb{A}^{d}$ is an affine variety, we may consider the analogous construction using the Gauss map $X \rightarrow \operatorname{Gr}(d, n)$, but this produces exactly the same thing, since the morphism $X \times \operatorname{Gr}(d, n) \rightarrow X \times \operatorname{Gr}(d+$ $1, n+1)$ given by $(x, V) \mapsto(x, K\langle(1 \times x)\rangle \oplus(0 \times V))$ is a closed embedding.

\subsection{Toric Varieties}

We review here some standard definitions and facts about toric varieties. For proofs, we refer the reader to [Ewald 96, Fulton 93, Miller and Sturmfels 05, Thaddeus 94].

A polyhedron in $\mathbb{Q}^{d}$ is a subset $P$ defined by finitely many weak affine inequalities, say $\sum_{j=1}^{d} a_{i j} x_{j} \geq b_{i}$. It is a polyhedral cone if the inequalities are linear, that is, all $b_{i}$ are zero. For simplicity we refer to polyhedral cones simply as cones. We also assume that all polyhedra are rational, meaning that all $a_{i j}$ and all $b_{i}$ are rational. A polyhedron is proper if it contains no affine linear subspace besides a point and is contained in no affine linear subspace besides $\mathbb{Q}^{d}$.

A face $F$ of $P$ is the locus on which equality holds in some fixed subset of the inequalities above. It is a facet if its affine linear span has codimension 1 in that of $P$. 
It is a vertex if it is a point. A proper cone in $\mathbb{Q}^{d}$ is simplicial if it has exactly $d$ facets. For any face $F \subset P$, the localization $P_{F}$ is the cone generated, as a semigroup, by the Minkowski difference $P-F$.

For $t \neq 0$, let $t P=\{t v \mid v \in P\}$; however, for $t=0$, by convention let $0 P$ denote the cone at infinity defined by the same inequalities as $P$, except with constant terms set to zero. The reason for this convention is that $\{(t, v) \in$ $\left.\mathbb{Q}_{+} \times \mathbb{Q}^{d} \mid v \in t P\right\}$ is then a cone in $\mathbb{Q}^{d+1}$, defined by the inequalities $\sum_{j=1}^{d} a_{i j} x_{j} \geq b_{i} x_{0}$ and $x_{0} \geq 0$. It is called the cone over $P$ and denoted by $C(P)$.

A torus is a product of finitely many copies of the multiplicative group of $K$. A toric variety is a normal variety on which a torus acts with finitely many orbits. There is a one-to-one correspondence between polyhedra with integer vertices and toric varieties that are projective over an affine variety, equipped with a lifting of the torus action to $\mathcal{O}(1)$. It is given as follows. For a polyhedron $P \subset \mathbb{Q}^{d}$, the semigroup algebra $K\left[C(P) \cap \mathbb{Z}^{d+1}\right]$ is graded by the zeroth coordinate. Let $X(P)=\operatorname{Proj} K\left[C(P) \cap \mathbb{Z}^{d+1}\right]$. This is a quasiprojective variety acted on by the torus $T=\operatorname{Spec} K\left[\mathbb{Z}^{d}\right]$. For example, if $P$ is already a cone, then $C(P)=\mathbb{Q}_{+} \times P$ and $X(P)=\operatorname{Proj} K\left[P \cap \mathbb{Z}^{d}\right]\left[x_{0}\right]=\operatorname{Spec} K\left[P \cap \mathbb{Z}^{d}\right]$, the affine toric variety usually associated to a cone. In general, $X(P)$ is projective over the affine variety $X(0 P)$, because $C(P) \cap\left(0 \times \mathbb{Q}^{d}\right)=0 P$.

Remark 2.5. A polyhedron $P$ is proper if and only if (a) the torus action on $X(P)$ is effective, and (b) $X(P)$ is not a direct product of a toric variety with a torus. So in light of Remark 2.3, there is no loss of generality, for the purposes of Nash blowing up, in assuming that $P$ is proper.

Remark 2.6. Any toric variety has a natural cover by open affine toric subvarieties. Indeed, $X(P)$ is covered by the affine varieties $X\left(P_{F}\right)$, where $F$ runs over the faces of $P$. If $P$ is proper, just the vertices are sufficient.

Remark 2.7. Define two cones to be equivalent if an element of $\operatorname{GL}(d, \mathbb{Z})$ takes one to the other. Then equivalent cones clearly lead to isomorphic toric varieties, with the torus action adjusted by the appropriate automorphism of $T$.

Remark 2.8. An affine toric variety $X(C)$, with $C$ proper, is smooth if and only if it is isomorphic to $\mathbb{A}^{d}$, or equivalently, if $C$ is equivalent to the orthant $\mathbb{Q}_{+}\left\langle e_{1}, \ldots, e_{d}\right\rangle$.

\subsection{Nash Blowups of Toric Varieties}

Now let $C$ be a cone in $\mathbb{Q}^{d}$. Let $H$ be the Hilbert basis of the semigroup $C \cap \mathbb{Z}^{d}$, that is, the set of indecomposable nonzero elements in the semigroup. This is the unique minimal set of generators of $C \cap \mathbb{Z}^{d}$. By Gordan's lemma [Fulton 93, Section 1.2, Proposition 1], $H$ is a finite set, say with $n$ elements. Let $M$ be the $n \times d$ integer matrix whose rows are the elements of $H$.

Let $S=\left\{h_{1}+\cdots+h_{d} \mid h_{i} \in H\right.$ linearly independent $\}$. Since $S$ is finite, its convex hull is a compact polyhedron Hull $S$. Hence the Minkowski sum $C+\operatorname{Hull} S$ is a polyhedron whose cone at infinity is $C$.

The following result is proved (in the language of fans) in [Gonzalez-Sprinberg 77b].

Theorem 2.9. The Nash blowup of $X(C)$ is $X(C+$ Hull $S)$.

Proof. Without loss of generality $C$ may be assumed proper. In this case $X(C)$ has a unique $T$-fixed point $q$.

Let $X=X(C)$. The Nash blowup of $X$ is plainly a toric variety, projective over $X$. It is therefore $X(P)$ for some polyhedron $P$ with $0 P=C$.

Such a polyhedron is uniquely determined by its cone at infinity $C$ and its vertices $v_{i}$. Indeed, the cone over $P$ is $C(P)=\mathbb{Q}_{+}\left\langle 0 \times C, 1 \times v_{i}\right\rangle$, and $P=C(P) \cap\left(1 \times \mathbb{Q}^{d}\right)$. So it suffices to show that, at the fixed points of the torus action on the Nash blowup, the weights of the torus action on $\mathcal{O}(1)$ are exactly the coordinates of the vertices of $C+$ Hull $S$.

Our choice of an embedding $X \subset \mathbb{A}^{n}$ will be the following canonical one. The surjection $\mathbb{Z}_{+}^{n} \rightarrow C \cap \mathbb{Z}^{d}$ sending the standard basis vectors to the rows of $M$ induces a surjection of algebras $K\left[\mathbb{Z}_{+}^{n}\right] \rightarrow K\left[C \cap \mathbb{Z}^{d}\right]$. The corresponding morphism $\operatorname{Spec} K\left[C \cap \mathbb{Z}^{d}\right] \rightarrow \operatorname{Spec} K\left[\mathbb{Z}_{+}^{n}\right]$ is the desired embedding.

Let $p$ be the base point of $X$ : the point such that $f(p)=1$ for every monomial $f \in K\left[C \cap \mathbb{Z}^{d}\right]$. The homomorphisms of algebras

$$
K\left[\mathbb{Z}_{+}^{n}\right] \rightarrow K\left[C \cap \mathbb{Z}^{d}\right] \rightarrow K\left[\mathbb{Z}^{d}\right] \rightarrow K
$$

where the last map sends every monomial to 1 , correspond to the inclusions of schemes

$$
\mathbb{A}^{n} \supset X \supset T \supset\{p\} .
$$

By Remark 2.4, we may consider the affine version of the Gauss map for this embedding. This is a rational map $G: X \rightarrow \operatorname{Gr}(d, n)$. We claim that $G(p)$ is the span of the columns of $M$. Indeed, in terms of variables $x_{1}, \ldots, x_{n}$ 
and $y_{1}, \ldots, y_{d}$, the homomorphism $K\left[\mathbb{Z}_{+}^{n}\right] \rightarrow K\left[\mathbb{Z}^{d}\right]$ is given by $x_{i} \mapsto \prod_{j} y_{j}^{m_{i j}}$. The parametric curve $y_{j}=1+$ $t \delta_{i j}$ in $T$ therefore maps to $x_{i}=(1+t)^{m_{i j}}$ in $\mathbb{A}^{n}$, so its derivative with respect to $t$ at 0 is $\left(m_{1 j}, \ldots, m_{n j}\right)$, the $j$ th column of $M$.

The coordinates of the Plücker embedding $\operatorname{Gr}(d, n) \rightarrow$ $\mathbb{P} \Lambda^{d} K^{n}$ are indexed by $d$-element subsets $I \subset\{1, \ldots, n\}$. This embedding is $T$-equivariant for the induced linear action of $T$ on $\mathbb{P} \Lambda^{d} K^{n}$. The $I$ th Plücker coordinate of $G(p)$ is the $I$ th minor of $M$. Hence $G(p)$ is contained in the linear subspace of $\mathbb{P} \Lambda^{d} K^{n}$ spanned by those coordinates $I$ for which the $I$ th minor of $M$ is nonzero.

Since the $T$-action on $\mathbb{P} \Lambda^{d} K^{n}$ is diagonal, the entire closure of the orbit of $G(p)$ must be contained in this subspace. Hence any fixed point in the closure of this orbit must be the $I$ th coordinate axis $e_{I}$ for some $I$ as above. If $I=\left\{i_{1}, \ldots, i_{d}\right\}$, then the nonvanishing of the $I$ th minor is equivalent to the linear independence of $h_{i_{1}}, \ldots, h_{i_{d}} \in H$, and the fiber of $\mathcal{O}(1)$ at this point is acted on with weight $h_{i_{1}}+\cdots+h_{i_{d}}$. That is, the weights at fixed points in this subspace are exactly the elements of $S$.

The closure of the graph of the Gauss map is clearly contained in $X \times \overline{T p}$, so its $T$-fixed points must be of the form $q \times e_{I}$, where $q$ is the unique fixed point in $X$, and $e_{I}$ is as above. The weights of $\mathcal{O}(1)$ at these points must therefore belong to $S$. The same is true for the normalization, since $\mathcal{O}(1)$ pulls back to an ample bundle there.

Consequently, $P$ is a polyhedron with $0 P=C$ and with vertices contained in $S$. Therefore $P \subset C+$ Hull $S$.

To establish equality, it suffices to show that every vertex in $C+$ Hull $S$ is the weight of some fixed point in the Nash blowup. For every vertex $v_{I}$ of $C+\operatorname{Hull} S$, there is a linear functional $f$ on $\mathbb{Q}^{d}$ whose restriction to $C+$ Hull $S$ takes on its minimum only at $v_{I}$. Hence its restriction to Hull $S$ takes on its minimum only at $v_{I}$, and its restriction to $C$ takes on its minimum only at 0 . The corresponding 1-parameter subgroup $\lambda(t): K^{\times} \rightarrow T$ therefore satisfies $\lim _{t \rightarrow 0} \lambda(t) \cdot G(p)=e_{I}$ and $\lim _{t \rightarrow 0} \lambda(t) \cdot p=q$. Hence $q \times e_{I} \in \overline{T \cdot(p \times G(p))}$, the closure of the graph of the Gauss map. A point in the normalization lying over $q \times e_{I}$ is acted on with the same weight. This completes the proof.

\section{RESOLUTION TREES}

We wish to consider whether a toric variety is desingularized by a finite sequence of Nash blowups. The Nash blowup is a local construction: that is, the Nash blowups of an open cover furnish an open cover of the Nash blowup. Hence it suffices to consider an affine toric variety $X(C)$. The Nash blowup of $X(C)$ is $X(C+\operatorname{Hull} S)$; by Remark 2.6, an open cover of this consists of the affine varieties $X\left((C+\operatorname{Hull} S)_{v}\right)$, where $v$ runs over the vertices of $C+$ Hull $S$. By Remark 2.8, $X(C+$ Hull $S)$ is smooth if and only if each localization $(C+\operatorname{Hull} S)_{v}$ is equivalent to the orthant under the action of $\operatorname{GL}(d, \mathbb{Z})$. If not, the Nash blowup can be repeated by applying the theorem to each cone $(C+\operatorname{Hull} S)_{v}$.

In other words, the process of iterating Nash blowups of $X(C)$ corresponds, via the toric dictionary, to the following algorithm in convex geometry:

(1) Given the cone $C$, find the Hilbert basis $H$ of $C \cap \mathbb{Z}^{d}$.

(2) Find

$S=\left\{h_{1}+\cdots+h_{d} \mid h_{i} \in H\right.$ linearly independent $\}$.

(3) Find the convex hull Hull $S$ (i.e., list its vertices, or list the inequalities defining it).

(4) Find the Minkowski sum $C+\operatorname{Hull} S$ (i.e., list its vertices and cone at infinity, or list the inequalities defining it).

(5) Find the localization $C^{\prime}=(C+\operatorname{Hull} S)_{v}$ of this Minkowski sum at each vertex $v$.

(6) Determine whether each such $C^{\prime}$ is equivalent to the orthant. If so, stop; if not, apply the entire algorithm to $C^{\prime}$.

Because each cone may give rise to several more in step (5), the algorithm branches. This can be expressed in terms of a graph as follows. Define the Nash blowup of a cone $C$ to be the finite set of cones of the form $(C+\operatorname{Hull} S)_{v}$, where $S$ is as in (2), and $v$ runs over the vertices of $C+$ Hull $S$. Then define the resolution tree of $C$ or $X(C)$ to be the unique rooted tree, with nodes labeled by cones in $\mathbb{Q}^{d}$, whose root is labeled by $C$, and where for every node, say labeled by $C^{\prime}$ :

(a) if $C^{\prime}$ is equivalent to the orthant, there are no edges beginning at $C^{\prime}$ (that is, $C^{\prime}$ is a leaf);

(b) otherwise, the edges beginning at $C^{\prime}$ connect it to nodes labeled by the cones $\left(C^{\prime}+\text { Hull } S^{\prime}\right)_{v^{\prime}}$ appearing in its Nash blowup.

It is then clear that $X(C)$ is desingularized by a finite number of Nash blowups if and only if its resolution tree is finite. It is equally clear that the latter property is amenable to computer investigation, using the algorithm 
above. We will report on this presently, but first, we explain how, in the 2-dimensional case, the situation can be completely understood.

\section{THE 2-DIMENSIONAL CASE}

Gonzalez-Sprinberg showed [Gonzalez-Sprinberg 77a, Gonzalez-Sprinberg 77b] that toric surfaces are desingularized by a finite sequence of (normalized) Nash blowups. This was later extended to arbitrary surfaces in [Hironaka 83] and [Gonzalez-Sprinberg 87, Spivakovsky 90]. In this section, we give an alternative proof of Gonzalez-Sprinberg's original result, emphasizing the role of Hirzebruch-Jung continued fractions. We begin by defining them and recalling their basic properties.

For integers $a_{1}, a_{2}, \ldots$, let

$$
\left[a_{1}, \ldots, a_{i}\right]=a_{1}-\frac{1}{a_{2}-\frac{1}{\ddots-\frac{1}{a_{i}}}} .
$$

We assume implicitly throughout that no denominator is zero; this is the case, for example, when $a_{i}>1$ for $i>1$.

Set $p_{-1}=0$ and $q_{0}=0$; set $p_{0}=1$ and $q_{1}=1$. Then recursively let

$$
p_{i}=a_{i} p_{i-1}-p_{i-2}, \quad q_{i}=a_{i} q_{i-1}-q_{i-2}
$$

for greater values of $i$.

Proposition 4.1. For $p_{i}, q_{i}$ as above, $\left[a_{1}, \ldots, a_{i}\right]=p_{i} / q_{i}$.

Proof. Using induction on $i$, we will prove the more general statement in which the $a_{i}$ are merely rational. The case $i=1$ is trivial. For $i>1$, assume that the statement holds for continued fractions of length $i-1$, and consider $\left[a_{1}, \ldots, a_{i-2}, a_{i-1}-1 / a_{i}\right]$. Let $P_{j}, Q_{j}$ be the numbers defined as above for this continued fraction. Then $P_{j}=p_{j}$ and $Q_{j}=q_{j}$ for $j<i-1$, and

$$
\begin{aligned}
{\left[a_{1}, \ldots, a_{i}\right] } & =\left[a_{1}, \ldots, a_{i-2}, a_{i-1}-1 / a_{i}\right]=\frac{P_{i-1}}{Q_{i-1}} \\
& =\frac{\left(a_{i-1}-1 / a_{i}\right) p_{i-2}-p_{i-3}}{\left(a_{i-1}-1 / a_{i}\right) q_{i-2}-q_{i-3}} \\
& =\frac{\left(a_{i-1} a_{i}-1\right) p_{i-2}-a_{i} p_{i-3}}{\left(a_{i-1} a_{i}-1\right) q_{i-2}-a_{i} q_{i-3}} \\
& =\frac{a_{i} p_{i-1}-p_{i-2}}{a_{i} q_{i-1}-q_{i-2}}=\frac{p_{i}}{q_{i}} .
\end{aligned}
$$

Proposition 4.2. For $i>0, p_{i-1} q_{i}-p_{i} q_{i-1}=1$.

Proof. Again use induction on $i$. The case $i=1$ is trivial. For $i>1$, by the induction hypothesis,

$$
\begin{aligned}
& p_{i-1} q_{i}-p_{i} q_{i-1} \\
& \quad=p_{i-1}\left(a_{i} q_{i-1}-q_{i-2}\right)-\left(a_{i} p_{i-1}-p_{i-2}\right) q_{i-1} \\
& \quad=-p_{i-1} q_{i-2}+p_{i-2} q_{i-1}=1 .
\end{aligned}
$$

Corollary 4.3. The fraction $p_{i} / q_{i}$ is in lowest terms.

Proposition 4.4. For $i<j$, the denominator of $\left[a_{i+1}, \ldots, a_{j}\right]$ as a fraction in lowest terms is $p_{i} q_{j}-p_{j} q_{i}$.

Proof. The case $i=j-1$ is covered by the previous proposition. Now proceed by descending induction on $i$. Let $\left[a_{i+1}, \ldots, a_{j}\right]=N_{i} / D_{i}$ in lowest terms, so that $D_{j-1}=1$ in particular. Take $N_{j}=1, D_{j}=0$ by convention. Then for all $i<j$ we have

$$
\begin{aligned}
{\left[a_{i+1}, \ldots, a_{j}\right] } & =a_{i+1}-\frac{1}{\left[a_{i+2}, \ldots, a_{j}\right]} \\
& =a_{i+1}-\frac{D_{i+1}}{N_{i+1}}=\frac{a_{i+1} N_{i+1}-D_{i+1}}{N_{i+1}}
\end{aligned}
$$

which is also in lowest terms. Hence $D_{i}=N_{i+1}$, and the $D_{i}$ satisfy the descending recurrence $D_{i}=a_{i+2} D_{i+1}-$ $D_{i+2}$ with initial conditions $D_{0}=0, D_{1}=1$. The same holds for

$$
\begin{aligned}
p_{i} q_{j}-p_{j} q_{i} & =\left(a_{i+2} p_{i+1}-p_{i+2}\right) q_{j}-p_{j}\left(a_{i+2} q_{i+1}-q_{i+2}\right) \\
& =a_{i+2}\left(p_{i+1} q_{j}-p_{j} q_{i+1}\right)-\left(p_{i+2} q_{j}-p_{j} q_{i+2}\right),
\end{aligned}
$$

which completes the proof.

Proposition 4.5. For any rational $x$, there exists a unique finite sequence of integers $a_{1}, \ldots, a_{k}$ with $a_{i}>1$ for $i>1$ such that $x=\left[a_{1}, \ldots, a_{k}\right]$.

Proof. For any such sequence and for any $i>1$, we have $\left[a_{i}, \ldots, a_{k}\right]>1$ by descending induction on $i$. If $x=$ $\left[a_{1}, \ldots, a_{k}\right]$, then $x=a_{1}-1 /\left[a_{2}, \ldots, a_{k}\right]$, so $a_{1}=\lceil x\rceil$ is uniquely determined. Then $1 /\left(a_{1}-x\right)=\left[a_{2}, \ldots, a_{k}\right]$, and hence $a_{2}$ is uniquely determined too. By induction, all the $a_{i}$ are uniquely determined.

As for existence, this can be established by iterating three operations: round up, subtract, and invert. That is, given $x_{1}=x$, let $a_{1}=\left\lceil x_{1}\right\rceil$, let $b_{1}=a_{1}-x_{1}$, and let $x_{2}=$ $1 / b_{1}$. Recursively, given $x_{i}$, let $a_{i}=\left\lceil x_{i}\right\rceil$, let $b_{i}=a_{i}-x_{i}$, and let $x_{i+1}=1 / b_{i}$. If $x_{i}=n_{i} / d_{i}$ is in lowest terms, then $x_{i+1}=d_{i} /\left(a_{i} d_{i}-n_{i}\right)$ is also in lowest terms, so $n_{i+1}=$ 
$d_{i}$. Since $x_{i}>1$ for $i>1$, the sequence of $d_{i}$ must be nonnegative and strictly decreasing, so eventually some $d_{i}$ will be equal to 1 (whereupon $x_{i+1}$ is undefined and the sequence ends). It is then easy to verify that $x=$ $\left[a_{1}, \ldots, a_{k}\right]$.

Corollary 4.6. If $a_{i}>1$ for $i>1$, then for $1<i \leq j$, the denominator of $\left[a_{i}, \ldots, a_{j}\right]$ is strictly less than that of $\left[a_{1}, \ldots, a_{j}\right]$.

Proof. The sequence of denominators is the strictly decreasing sequence $d_{i}$ appearing in the proof of the previous proposition.

Proposition 4.7. If $a_{i}>1$ for $i>1$, then for all $i<j$, the denominator of $\left[a_{1}, \ldots, a_{i}\right]$ is strictly less than that of $\left[a_{1}, \ldots, a_{j}\right]$.

Proof. The denominators are exactly the $q_{i}$, so this is equivalent to showing that the $q_{i}$ are strictly increasing, which is proved by induction on $i$ : if $q_{i-1}-q_{i-2}>0$, then

$$
\begin{aligned}
q_{i}-q_{i-1} & =a_{i} q_{i-1}-q_{i-2}-q_{i-1} \\
& =\left(a_{i}-1\right) q_{i-1}+q_{i-1}-q_{i-2}>0 .
\end{aligned}
$$

Corollary 4.8. If $a_{i}>1$ for $i>1$, then for all $1<i<j<$ $k$, the denominator of $\left[a_{i}, \ldots, a_{j}\right]$ is strictly less than that of $\left[a_{1}, \ldots, a_{k}\right]$.

Proof. Combine the last two results.

Now let $C$ be a proper cone in $\mathbb{Q}^{2}$. It can be placed in a standard form as follows.

Proposition 4.9. There exists an element of $\mathrm{SL}(2, \mathbb{Z})$ taking $C$ to $\mathbb{Q}_{+}\langle(1,0),(p, q)\rangle$ with $0 \leq p<q$ and $p, q$ coprime: that is, a cone in the first quadrant subtending an angle between $45^{\circ}$ and $90^{\circ}$.

Proof. Any proper cone in $\mathbb{Q}^{2}$ has two facets or edges. Let $(a, b) \in \mathbb{Z}^{2}$ be the smallest nonzero integer point along the clockwise edge. Then $a$ is coprime to $b$, say $a c+b d=1$, and $\left(\begin{array}{rr}c & d \\ -b & a\end{array}\right) \in \mathrm{SL}(2, \mathbb{Z})$ takes $C$ to a cone whose clockwise edge is along the positive $x$-axis and hence is contained in the first and second quadrants. Let $(e, f)$ be the smallest nonzero integer point along the counterclockwise edge. Since $f>0$, there exists an integer $g$ such that $0 \leq e+g f<f$. Then $\left(\begin{array}{ll}1 & g \\ 0 & 1\end{array}\right) \in \mathrm{SL}(2, \mathbb{Z})$ takes this cone to $\mathbb{Q}_{+}\langle(1,0),(e+g f, f)\rangle$, which satisfies the desired properties.

In light of the last proposition, we may assume $C=$ $\mathbb{Q}_{+}\langle(1,0),(p, q)\rangle$ for coprime $p, q$ with $0 \leq p<q$. As in Section 2, the intersection $C \cap \mathbb{Z}^{2}$ is an additive semigroup with a finite Hilbert basis $H$. In this simple case, the Hilbert basis may be explicitly described.

Proposition 4.10. If $p / q=\left[a_{1}, \ldots, a_{k}\right]$, then $H=$ $\left\{v_{0}, \ldots, v_{k}\right\}$, where $v_{i}=\left(p_{i}, q_{i}\right) \in \mathbb{Z}^{2}$.

Proof. Since $p_{i-1} q_{i}-p_{i} q_{i-1}=1$, the slopes of the rays through the $v_{i}$ are strictly increasing, and the lattice points in $\mathbb{Q}_{+}\left\langle v_{i-1}, v_{i}\right\rangle$ are all integral linear combinations of $v_{i-1}$ and $v_{i}$. This fan of subcones covers the entire cone, so any nonzero indecomposable element must be one of the $v_{i}$.

Conversely, since the $q_{i}$ are strictly increasing, if any $v_{i}$ can be nontrivially expressed as an integral combination of indecomposable elements, those elements must belong to $\left\{v_{0}, \ldots, v_{i-1}\right\}$. But this is absurd, since those elements subtend a smaller cone that does not contain $v_{i}$.

Now, as in Sections 2 and 3, let $S=\left\{v_{i}+v_{j} \mid 0 \leq i<\right.$ $j \leq k\}$.

Proposition 4.11. If $S^{\prime}=\left\{v_{i}+v_{i+1} \mid 0 \leq i<k\right\}$, then $C+\operatorname{Hull} S^{\prime}=C+\operatorname{Hull} S$.

Proof. One inclusion is trivial. For the other, it suffices to show that $v_{i}+v_{j} \in C+\operatorname{Hull} S^{\prime}$ for $0<i+1<j \leq k$. In fact, we will show that $v_{i}+v_{j}$ is in the even smaller set $C+\operatorname{Hull}\left\{v_{i}+v_{i+1}, v_{j-1}+v_{j}\right\}$. This is bounded by three lines, so it suffices to show that $v_{i}+v_{j}$ is on the correct side of each.

First, consider the line joining $v_{i}+v_{i+1}$ and $v_{j-1}+v_{j}$. To simplify the notation, let $\left\langle(x, y),\left(x^{\prime}, y^{\prime}\right)\right\rangle=x y^{\prime}-x^{\prime} y$, which is positive if and only if $\left(x^{\prime}, y^{\prime}\right)$ is counterclockwise from $(x, y)$.

By Proposition 4.4, $\left\langle v_{i}, v_{j}\right\rangle>0$ for $i<j$, and hence $\left\langle v_{i}+v_{i+1}, v_{j-1}+v_{j}\right\rangle>0$ too. For any two points $u_{1}, u_{2} \in \mathbb{Q}^{2}$, the affine linear functional $f(u)=\left\langle u_{1}, u\right\rangle+$ $\left\langle u, u_{2}\right\rangle-\left\langle u_{1}, u_{2}\right\rangle$ vanishes on the line joining $u_{1}$ and $u_{2}$. Let $u_{1}=v_{i}+v_{i+1}$ and $u_{2}=v_{j-1}+v_{j}$; then $f(0)=$ $-\left\langle v_{i}+v_{i+1}, v_{j-1}+v_{j}\right\rangle<0$. A brief calculation shows that

$f\left(v_{i}+v_{j}\right)=\left\langle v_{i}, v_{j}\right\rangle-\left\langle v_{i}, v_{i+1}\right\rangle-\left\langle v_{i+1}, v_{j-1}\right\rangle-\left\langle v_{j-1}, v_{j}\right\rangle$. 
By Proposition 4.2, $\left\langle v_{i}, v_{i+1}\right\rangle=\left\langle v_{j-1}, v_{j}\right\rangle=1$, and by Proposition 4.4 and Corollary 4.8 ,

$$
\left\langle v_{i}, v_{j}\right\rangle>\left\langle v_{i+1}, v_{j}\right\rangle>\left\langle v_{i+1}, v_{j-1},\right\rangle
$$

so

$$
\left\langle v_{i}, v_{j}\right\rangle \geq\left\langle v_{i+1}, v_{j-1}\right\rangle+2 \text { and } f\left(v_{i}+v_{j}\right) \geq 0 .
$$

Therefore $v_{i}+v_{j}$ is on the correct side of the line.

Next, consider the line through $v_{i}+v_{i+1}$ with slope $v_{0}$. For $v_{i}+v_{j}$ to be on the correct side of the line, we need $\left(v_{i}+v_{j}\right)-\left(v_{i}+v_{i+1}\right)=v_{j}-v_{i+1}$ to be counterclockwise from $v_{0}$, that is, $\left\langle v_{0}, v_{j}\right\rangle>\left\langle v_{0}, v_{i+1}\right\rangle$. Since $j \geq i+1$, this follows from Propositions 4.4 and 4.7.

The case of the third line is similar.

So if $i$ and $j$ are not consecutive, then $v_{i}+v_{j}$ is inessential to the shape of $C+$ Hull $S$. However, if they are consecutive, then the opposite is true, in the following sense.

Proposition 4.12. The boundary of $C+$ Hull $S$ consists of the line segments joining $v_{i-1}+v_{i}$ and $v_{i}+v_{i+1}$ for $0<i<k$, together with two rays starting at $v_{0}+v_{1}$ and $v_{k-1}+v_{k}$ and pointing in the directions $v_{0}$ and $v_{k}$, respectively.

Proof. Since $v_{0}=(1,0)$, it suffices to show that the slopes of these line segments are positive (or possibly $+\infty$ ) and weakly decreasing, and finally, that the slope of $v_{k}$ is no greater than the slope of the last line segment.

The line segments in question have direction $v_{i+1}-$ $v_{i-1}$, so it must be shown that

$$
\frac{q_{i+1}-q_{i-1}}{p_{i+1}-p_{i-1}} \geq \frac{q_{i+2}-q_{i}}{p_{i+2}-p_{i}} .
$$

Cross-multiplying and using Proposition 4.2 shows this to be equivalent to $p_{i-1} q_{i+2}-p_{i+2} q_{i-1} \geq 1$, which follows from Proposition 4.4. For the last part of the claim, it must be shown that

$$
\frac{q_{k}-q_{k-2}}{p_{k}-p_{k-2}} \geq \frac{p_{k}}{q_{k}}
$$

which follows from Proposition 4.4, again after crossmultiplying.

We have shown that the vertices of $C+\operatorname{Hull} S$ are all of the form $v_{i}+v_{i+1}$ (although not all $v_{i}+v_{i+1}$ need be vertices: see Figure 1). By Proposition 4.9, the localization of $C$ at any such vertex can be taken by an element of $\mathrm{SL}(2, \mathbb{Z})$ to a cone of the form $\mathbb{Q}_{+}\left\langle(1,0),\left(p^{\prime}, q^{\prime}\right)\right\rangle$ for $p, q^{\prime}$ coprime and $0 \leq p^{\prime}<q^{\prime}$.

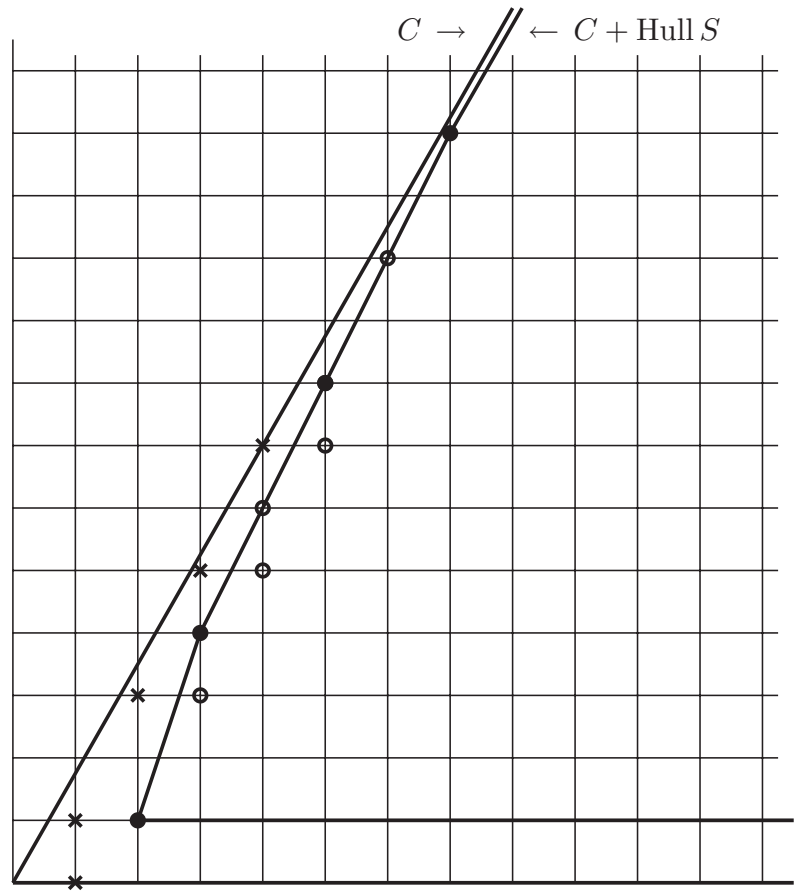

FIGURE 1. The case $p / q=4 / 7: C=\mathbb{Q}_{+}\langle(1,0),(4,7)\rangle$, $H=\{\times\}, S=\{\bullet, \circ\}, S^{\prime}=\{\bullet\}$.

Proposition 4.13. Unless $p=q-1$, every localized cone satisfies $q^{\prime}<q$.

Proof. There are two cases: the internal case where $0<$ $i<k-1$ and the external case where $i=0$ or $i=k-1$.

In the internal case, the two edges of the localized cone are along $\left(v_{i-1}+v_{i}\right)-\left(v_{i}+v_{i+1}\right)=v_{i-1}-$ $v_{i+1}$ and $\left(v_{i+1}+v_{i+2}\right)-\left(v_{i}+v_{i+1}\right)=v_{i+2}-v_{i}$. Hence $q^{\prime}=\left\langle v_{i-1}-v_{i+1}, v_{i+2}-v_{i}\right\rangle=\left\langle v_{i-1}, v_{i+2}\right\rangle-3$ by Proposition 4.2. By Proposition 4.4, $\left\langle v_{i-1}, v_{i+2}\right\rangle$ is the denominator of $\left[a_{i}, a_{i+1}, a_{i+2}\right]$, which by Corollary 4.8 is strictly less than $q$.

In the external case, consider first $i=0$. The two edges of the localized cone are along $v_{0}$ and $\left(v_{1}+v_{2}\right)-\left(v_{0}+\right.$ $\left.v_{1}\right)=v_{2}-v_{0}$, so $q^{\prime}=\left\langle v_{0}, v_{2}-v_{0}\right\rangle=\left\langle v_{0}, v_{2}\right\rangle$, which is the denominator of $\left[a_{1}, a_{2}\right]$. Again by Corollary 4.8 , this is strictly less than $q$ unless $p / q=\left[a_{1}, a_{2}\right]$, so that $k=2$. If so, the condition $0 \leq p<q$ implies $a_{1}=1$, so $p / q=$ $\left(a_{2}-1\right) / a_{2}$ and $p=q-1$.

Likewise, when $i=k-1$, the two edges of the localized cone are along $v_{k-2}-v_{k}$ and $v_{k}$, so $q^{\prime}=$ $\left\langle v_{k-2}-v_{k}, v_{k}\right\rangle=\left\langle v_{k-2}, v_{k}\right\rangle$, which is the denominator of $\left[a_{k-1}, a_{k}\right]$. Again, this is strictly less than $q$ unless $p / q=$ $\left[a_{k-1}, a_{k}\right]$, so that $k=2$. Hence $p=q-1$ again. 
We are now in a position to prove GonzalezSprinberg's result [Gonzalez-Sprinberg 77a, GonzalezSprinberg $77 \mathrm{~b}]$.

Theorem 4.14. Any toric surface is desingularized by a finite number of Nash blowups.

Proof. The question is local, so it suffices to consider an affine toric surface corresponding to a cone $\mathbb{Q}_{+}\langle(1,0),(p, q)\rangle$, with $0 \leq p<q$ and $p, q$ coprime. This surface is smooth if and only if $q=1$, for only then will the Hilbert basis consist of exactly two elements. The previous proposition shows that $q$ is strictly decreasing under Nash blowups except at external vertices for $p=q-1$. In this case, a direct calculation shows that both external vertices have $p^{\prime} / q^{\prime}=(q-2) / q$, so the denominator will strictly decrease at the next step.

\section{A METHOD FOR ENUMERATING SIMPLICIAL CONES}

In dimension greater than 2 , we have no general results on the resolution of toric varieties by Nash blowups. However, using a computer, we have carried out an extensive investigation of 3- and 4-dimensional examples. Our primary focus is on simplicial cones, which correspond in the toric dictionary to affine toric orbifolds. But, as we will see, more general cones appear in the Nash blowups of simplicial cones and must be treated as part of the recursions.

We shall begin, then, by explaining how the simplicial cones of a given dimension $d$, or rather their equivalence classes under the action of $\mathrm{GL}(d, \mathbb{Z})$, can be systematically enumerated.

Any proper cone $C \subset \mathbb{Q}^{d}$ is defined by finitely many linear inequalities with integer coefficients, say $\sum_{j=1}^{d} a_{i j} x_{j} \geq 0$ for $1 \leq i \leq m$. Without loss of generality, assume that (i) no inequality is redundant in that it follows from the others; and (ii) for each fixed $i$, the $a_{i j}$ are coprime. The $m \times d$ integer matrix $A=\left(a_{i j}\right)$ is then called a presentation of $C$. It is unique modulo the left action of the group $S_{m}$ of permutation matrices. To classify cones modulo $\mathrm{GL}(d, \mathbb{Z})$, then, is equivalent to classifying integer matrices $A$ satisfying (i) and (ii) modulo $S_{m} \times \mathrm{GL}(d, \mathbb{Z})$ acting on the left and right. This is accomplished in practice using the following invariant.

For a cone $C$ with presentation $A$, let $\Lambda \subset \mathbb{Z}^{d}$ be the subgroup generated by the rows of $A$. Define the index $I(C) \in \mathbb{Z}_{+}$to be the index of $\Lambda$ as a subgroup of $\mathbb{Z}^{d}$. (This is the order of the orbifold group at the fixed point of the torus action.) Also, if $C^{*}=\left\{u \in \mathbb{Q}^{d} \mid \forall v \in C, u \cdot v \geq 0\right\}$ is the dual cone, define the dual index $I^{*}(C)$ to be $I\left(C^{*}\right)$. Clearly $I(C)$ and $I^{*}(C)$ are invariant under the $\mathrm{GL}(d, \mathbb{Z})$ action.

It is, of course, nettlesome to decide whether a given matrix satisfies the nonredundancy condition (i). But in the simplicial case it is easy: a presentation $A$ of a simplicial cone is exactly a nonsingular square integer matrix satisfying (ii). As such, $A$ can be taken by the right action of $\operatorname{GL}(d, \mathbb{Z})$ into Hermite normal form [Schrijver 86, 4.1]. This means that there exists $B \in \mathrm{GL}(d, \mathbb{Z})$ such that $A B$ is lower triangular, with nonnegative entries, and each row has a unique greatest entry located on the diagonal. Furthermore, since the entries in any given row of $A$ are coprime, the same is true of $A B$. These facts may be summarized as follows.

Proposition 5.1. Every simplicial cone $C$ is equivalent to one with a presentation $A$ in Hermite normal form, each of whose rows has coprime entries.

Corollary 5.2. There are finitely many equivalence classes of simplicial cones of dimension $d$ and index I.

Proof. In the simplicial case, $I(C)=|\operatorname{det} A|$, so if $A$ is in Hermite normal form, its diagonal entries multiply to $I$. Hence there are only finitely many choices for the diagonal entries of $A$, and so for the subdiagonal entries as well.

For a fixed value of $I$, it is now practical to enumerate the equivalence classes of cones $C$ using Proposition 5.1. Indeed, two matrices $A$ and $A^{\prime}$ are equivalent if and only if $S A T=A^{\prime}$ for some $S \in S_{d}$ and $T \in \mathrm{GL}(d, \mathbb{Z})$. Detecting this is a tractable problem for small $d$, since one can consider $A^{-1} S A^{\prime}$ for all $S \in S_{d}$ and see whether any of them is an integer matrix.

In this manner, the numbers $T_{d}(I)$ of equivalence classes of $d$-dimensional cones of index $I$ were determined with a computer for small values of $I$. These numbers are presented in Table 1 for $d=3$ and in Table 2 for $d=4$. A list of explicit representatives for each of these equivalence classes, for the first few values of $I$, is given in Table 3 for $d=3$ and in Table 4 for $d=4$. Many cones are reducible to a direct sum of cones of lower dimension; in such cases, the direct sum in question is shown in the right-hand column of Tables 3 and 4. By Remark 2.3, the Nash blowup of a direct sum of cones is the direct sum of their Nash blowups, so only irreducible cones are interesting for our purposes. 


\begin{tabular}{|c|c|c|c|c|c|c|c|c|c|c|c|c|c|}
\hline$I$ & $T_{3}(I)$ & $I$ & $T_{3}(I)$ & $I$ & $T_{3}(I)$ & $I$ & $T_{3}(I)$ & $I$ & $T_{3}(I)$ & $I$ & $T_{3}(I)$ & $I$ & $T_{3}(I)$ \\
\hline 1 & 1 & 31 & 182 & 61 & 662 & 91 & 1679 & 121 & 2705 & 151 & 3902 & 181 & 5582 \\
\hline 2 & 2 & 32 & 227 & 62 & 693 & 92 & 1643 & 122 & 2583 & 152 & 4591 & 182 & 6595 \\
\hline 3 & 4 & 33 & 241 & 63 & 898 & 93 & 1715 & 123 & 2951 & 153 & 4872 & 183 & 6425 \\
\hline 4 & 7 & 34 & 221 & 64 & 838 & 94 & 1551 & 124 & 2919 & 154 & 4777 & 184 & 6633 \\
\hline 5 & 8 & 35 & 277 & 65 & 883 & 95 & 1825 & 125 & 3072 & 155 & 4717 & 185 & 6667 \\
\hline 6 & 11 & 36 & 311 & 66 & 915 & 96 & 2051 & 126 & 3484 & 156 & 5298 & 186 & 6729 \\
\hline 7 & 14 & 37 & 254 & 67 & 794 & 97 & 1634 & 127 & 2774 & 157 & 4214 & 187 & 6695 \\
\hline 8 & 21 & 38 & 273 & 68 & 925 & 98 & 1846 & 128 & 3211 & 158 & 4293 & 188 & 6555 \\
\hline 9 & 23 & 39 & 329 & 69 & 965 & 99 & 2110 & 129 & 3239 & 159 & 4875 & 189 & 7872 \\
\hline 10 & 25 & 40 & 381 & 70 & 1057 & 100 & 2135 & 130 & 3445 & 160 & 5555 & 190 & 7177 \\
\hline 11 & 28 & 41 & 308 & 71 & 888 & 101 & 1768 & 131 & 2948 & 161 & 5047 & 191 & 6208 \\
\hline 12 & 43 & 42 & 393 & 72 & 1206 & 102 & 2099 & 132 & 3852 & 162 & 5283 & 192 & 7942 \\
\hline 13 & 38 & 43 & 338 & 73 & 938 & 103 & 1838 & 133 & 3485 & 163 & 4538 & 193 & 6338 \\
\hline 14 & 45 & 44 & 411 & 74 & 975 & 104 & 2227 & 134 & 3105 & 164 & 5021 & 194 & 6435 \\
\hline 15 & 59 & 45 & 476 & 75 & 1254 & 105 & 2617 & 135 & 4114 & 165 & 6211 & 195 & 8569 \\
\hline 16 & 66 & 46 & 391 & 76 & 1143 & 106 & 1961 & 136 & 3709 & 166 & 4731 & 196 & 7799 \\
\hline 17 & 60 & 47 & 400 & 77 & 1219 & 107 & 1980 & 137 & 3220 & 167 & 4760 & 197 & 6600 \\
\hline 18 & 76 & 48 & 546 & 78 & 1257 & 108 & 2561 & 138 & 3763 & 168 & 6589 & 198 & 8292 \\
\hline 19 & 74 & 49 & 477 & 79 & 1094 & 109 & 2054 & 139 & 3314 & 169 & 5187 & 199 & 6734 \\
\hline 20 & 101 & 50 & 508 & 80 & 1434 & 110 & 2499 & 140 & 4454 & 170 & 5783 & 200 & 8624 \\
\hline 21 & 107 & 51 & 543 & 81 & 1350 & 111 & 2417 & 141 & 3853 & 171 & 6046 & 201 & 7727 \\
\hline 22 & 99 & 52 & 561 & 82 & 1189 & 112 & 2702 & 142 & 3479 & 172 & 5511 & 202 & 6969 \\
\hline 23 & 104 & 53 & 504 & 83 & 1204 & 113 & 2204 & 143 & 3985 & 173 & 5104 & 203 & 7933 \\
\hline 24 & 153 & 54 & 610 & 84 & 1644 & 114 & 2601 & 144 & 4668 & 174 & 5907 & 204 & 8866 \\
\hline 25 & 135 & 55 & 643 & 85 & 1473 & 115 & 2639 & 145 & 4141 & 175 & 6566 & 205 & 8153 \\
\hline 26 & 135 & 56 & 703 & 86 & 1305 & 116 & 2565 & 146 & 3675 & 176 & 6370 & 206 & 7245 \\
\hline 27 & 163 & 57 & 671 & 87 & 1507 & 117 & 2908 & 147 & 4584 & 177 & 6017 & 207 & 8762 \\
\hline 28 & 183 & 58 & 609 & 88 & 1625 & 118 & 2419 & 148 & 4113 & 178 & 5429 & 208 & 8774 \\
\hline 29 & 160 & 59 & 620 & 89 & 1380 & 119 & 2809 & 149 & 3800 & 179 & 5460 & 209 & 8311 \\
\hline 30 & 211 & 60 & 878 & 90 & 1828 & 120 & 3483 & 150 & 4894 & 180 & 7712 & 210 & 10273 \\
\hline
\end{tabular}

TABLE 1. Number of $\mathrm{GL}(3, \mathbb{Z})$-equivalence classes of simplicial cones in three dimensions.

\section{RESULTS OF COMPUTER INVESTIGATIONS}

We are now in a position to describe the empirical data obtained with a computer. Our program, resolve, was written in the language $\mathrm{C}++$ and relies heavily on the Boost open-source software libraries for $\mathrm{C}++$, especially the linear algebra library uBLAS of Joerg Walter and Mathias Koch. ${ }^{1}$

One function of the program is to enumerate the simplicial cones of a given dimension and index, as described in the previous section. However, the primary function of resolve is to implement the algorithm of Section 3 for carrying out the Nash blowup and to perform it it-

\footnotetext{
${ }^{1}$ Boost $\mathrm{C}++$ software library available at http://www.boost.org/ doc/libs. Our source code, as well as extensive tables of output, are available at http://www.math.columbia.edu/ thaddeus/nash. html.
}

eratively. The $\mathrm{C}++$ program often invokes the external programs $4 \mathrm{ti} 2,{ }^{2} \operatorname{lrs},{ }^{3}$ and qhull, ${ }^{4}$ which perform isolated parts of the computation. Specifically, 4 ti2 is used in Step 1 to find the Hilbert basis of $C \cap \mathbb{Z}^{n}$, while lrs is used in Steps 3 and 4 to determine the vertices of the polyhedron $C+\operatorname{Hull} S$, and the localization at each vertex; qhull is also used in Step 3 to simplify the determination of the convex hull. Because of the intensive nature of the latter computation, Step 3 requires by far the most computing time.

We used resolve to find Nash resolutions (that is, finite resolution trees of Nash blowups) for all 16023 dimensional simplicial cones with $I \leq 27$ and all 201

\footnotetext{
${ }^{2}$ Available at http://www.4ti2.de.

${ }^{3}$ Software available at http://jeff.cs.mcgill.ca/ avis/C/lrs.html.

${ }^{4}$ Software available at http://www.qhull.org/.
} 


\begin{tabular}{|c|c|c|c|c|c|c|c|c|c|}
\hline$I$ & $T_{4}(I)$ & $I$ & $T_{4}(I)$ & $I$ & $T_{4}(I)$ & $I$ & $T_{4}(I)$ & $I$ & $T_{4}(I)$ \\
\hline 1 & 1 & 11 & 101 & 21 & 788 & 31 & 1550 & 41 & 3399 \\
\hline 2 & 3 & 12 & 262 & 22 & 851 & 32 & 3083 & 42 & 7441 \\
\hline 3 & 7 & 13 & 154 & 23 & 682 & 33 & 2622 & 43 & 3891 \\
\hline 4 & 16 & 14 & 264 & 24 & 1778 & 34 & 2799 & 44 & 7172 \\
\hline 5 & 18 & 15 & 337 & 25 & 1037 & 35 & 2969 & 45 & 7652 \\
\hline 6 & 37 & 16 & 476 & 26 & 1338 & 36 & 5403 & 46 & 6552 \\
\hline 7 & 36 & 17 & 305 & 27 & 1530 & 37 & 2544 & 47 & 5012 \\
\hline 8 & 83 & 18 & 657 & 28 & 2123 & 38 & 3821 & 48 & 12605 \\
\hline 9 & 85 & 19 & 409 & 29 & 1288 & 39 & 4155 & 49 & 6512 \\
\hline 10 & 116 & 20 & 894 & 30 & 3006 & 40 & 6591 & 50 & 10047 \\
\hline
\end{tabular}

TABLE 2. Number of $\operatorname{GL}(4, \mathbb{Z})$-equivalence classes of simplicial cones in four dimensions.

\begin{tabular}{|c|c|c|c|c|c|c|c|c|c|c|c|c|}
\hline Name & $I$ & $I^{*}$ & Prese & ntation & & Reducibility & Name & $I$ & $I^{*}$ & Presentatic & & Reducibility \\
\hline$C_{1,1}$ & 1 & 1 & $\left(e_{1}\right.$ & $e_{2}$ & $\left.e_{3}\right)$ & $A \oplus A \oplus A$ & $C_{5,4}$ & 5 & 25 & $\left(e_{1}, \quad e_{2}\right.$ & $(1,1,5))$ & \\
\hline$C_{2,1}$ & 2 & 2 & $\left(e_{1}\right.$ & $e_{2}$ & $(0,1,2))$ & $B_{2,1} \oplus A$ & $C_{5,5}$ & 5 & 25 & $\left(e_{1}, \quad e_{2}\right.$ & $(1,2,5))$ & \\
\hline$C_{2,2}$ & 2 & 4 & $\left(e_{1}\right.$ & $e_{2}$ & $(1,1,2))$ & & $C_{5,6}$ & 5 & 25 & $\left(e_{1}, \quad e_{2}\right.$, & $(2,2,5))$ & \\
\hline$C_{3,1}$ & 3 & 3 & $\left(e_{1}\right.$ & $e_{2}$ & $(0,1,3))$ & $B_{3,1} \oplus A$ & $C_{5,7}$ & 5 & 25 & $\left(e_{1}, \quad e_{2}\right.$ & $(2,4,5))$ & \\
\hline$C_{3,2}$ & 3 & 3 & $\left(e_{1}\right.$ & $e_{2}$ & $(0,2,3))$ & $B_{3,2} \oplus A$ & $C_{5,8}$ & 5 & 25 & $\left(e_{1}, \quad e_{2}\right.$ & $(4,4,5))$ & \\
\hline$C_{3,3}$ & 3 & 9 & $\left(e_{1}\right.$ & $e_{2}$ & $(1,1,3))$ & & $C_{6,1}$ & 6 & 6 & $\left(e_{1}, \quad e_{2}\right.$ & $(0,1,6))$ & $B_{6,1} \oplus A$ \\
\hline$C_{3,4}$ & 3 & 9 & $\left(e_{1}\right.$ & $e_{2}$ & $(2,2,3))$ & & $C_{6,2}$ & 6 & 6 & $\left(e_{1}\right.$, & $(0,5,6))$ & $B_{6,2} \oplus A$ \\
\hline$C_{4,1}$ & 4 & 4 & $\left(e_{1}\right.$ & $e_{2}$ & $(0,1,4))$ & $B_{4,1} \oplus A$ & $C_{6,3}$ & 6 & 36 & $\left(e_{1}\right.$ & $(1,1,6))$ & \\
\hline$C_{4,2}$ & 4 & 4 & $\left(e_{1}\right.$, & $e_{2}$ & $(0,3,4))$ & $B_{4,2} \oplus A$ & $C_{6,4}$ & 6 & 18 & $\left(e_{1}, \quad e_{2}\right.$, & $(1,2,6))$ & \\
\hline$C_{4,3}$ & 4 & 16 & $\left(e_{1}\right.$ & $e_{2}$ & $(1,1,4))$ & & $C_{6,5}$ & 6 & 12 & $\left(e_{1}, \quad e_{2}\right.$, & $(1,3,6))$ & \\
\hline$C_{4,4}$ & 4 & 8 & $\left(e_{1}\right.$ & $e_{2}$ & $(1,2,4))$ & & $C_{6,6}$ & 6 & 6 & $\left(e_{1}, \quad e_{2}\right.$ & $(2,3,6))$ & \\
\hline$C_{4,5}$ & 4 & 8 & $\left(e_{1}\right.$ & $e_{2}$ & $(2,3,4))$ & & $C_{6,7}$ & 6 & 18 & $\left(e_{1}, \quad e_{2}\right.$ & $(2,5,6))$ & \\
\hline$C_{4,6}$ & 4 & 16 & $\left(e_{1}\right.$ & $e_{2}$ & $(3,3,4))$ & & $C_{6,8}$ & 6 & 6 & $\left(e_{1}, \quad e_{2}\right.$ & $(3,4,6))$ & \\
\hline$C_{4,7}$ & 4 & 2 & $\left(e_{1}\right.$ & $(1,2,0)$ & $(1,0,2))$ & & $C_{6,9}$ & 6 & 12 & $\left(e_{1}, \quad e_{2}\right.$ & $(3,5,6))$ & \\
\hline$C_{5,1}$ & 5 & 5 & $\left(e_{1}\right.$ & $e_{2}$ & $(0,1,5))$ & $B_{5,1} \oplus A$ & $C_{6,10}$ & 6 & 18 & $\left(e_{1}, \quad e_{2}\right.$ & $(4,5,6))$ & \\
\hline$C_{5,2}$ & 5 & 5 & $\left(e_{1}\right.$ & $e_{2}$ & $(0,2,5))$ & $B_{5,2} \oplus A$ & $C_{6,11}$ & 6 & 36 & $\left(e_{1}, \quad e_{2}\right.$, & $(5,5,6))$ & \\
\hline$C_{5,3}$ & 5 & 5 & $\left(e_{1}\right.$ & $e_{2}$ & $(0,4,5))$ & $B_{5,3} \oplus A$ & & & & & & \\
\hline
\end{tabular}

TABLE 3. Classification of simplicial cones in three dimensions.

4-dimensional simplicial cones with $I \leq 8$, following the classification. A few higher-dimensional cones were also resolved, but these required considerably more time. To improve efficiency, resolve ceases searching deeper in a resolution tree whenever it reaches a simplicial cone with $I$ strictly less than the initial value, since this has been resolved already. However, many nonsimplicial cones are encountered in the process of resolving simplicial cones, as are simplicial cones with equal or greater values of $I$.

Table 5 presents the Nash resolutions of all irreducible 3-dimensional simplicial cones of index $I \leq 4$. Likewise, Table 6 presents the Nash resolutions of all irreducible 4-dimensional simplicial cones of index $I \leq 4$. In both tables, each line displays the rows of a presentation of a single cone. The index $I$ and dual index $I^{*}$ are shown in brackets at the right. The first line in each block of text represents the original cone being resolved. The singly indented lines below it show the cones appearing in the Nash blowup of that cone. Subsequent to each of those, the doubly indented lines show the cones appearing in the Nash blowups of those cones.

Figure 2 depicts the resolution trees of all 3 dimensional irreducible simplicial cones of index $I \leq 6$. Likewise, Figure 3 depicts the resolution trees of all but one of the 4-dimensional irreducible simplicial cones of index $I \leq 5$. (One cone of index 5 , namely $D_{5,14}$, has an enormous resolution tree and has been omitted.) To avoid redundancy, each tree has been pruned of subtrees 


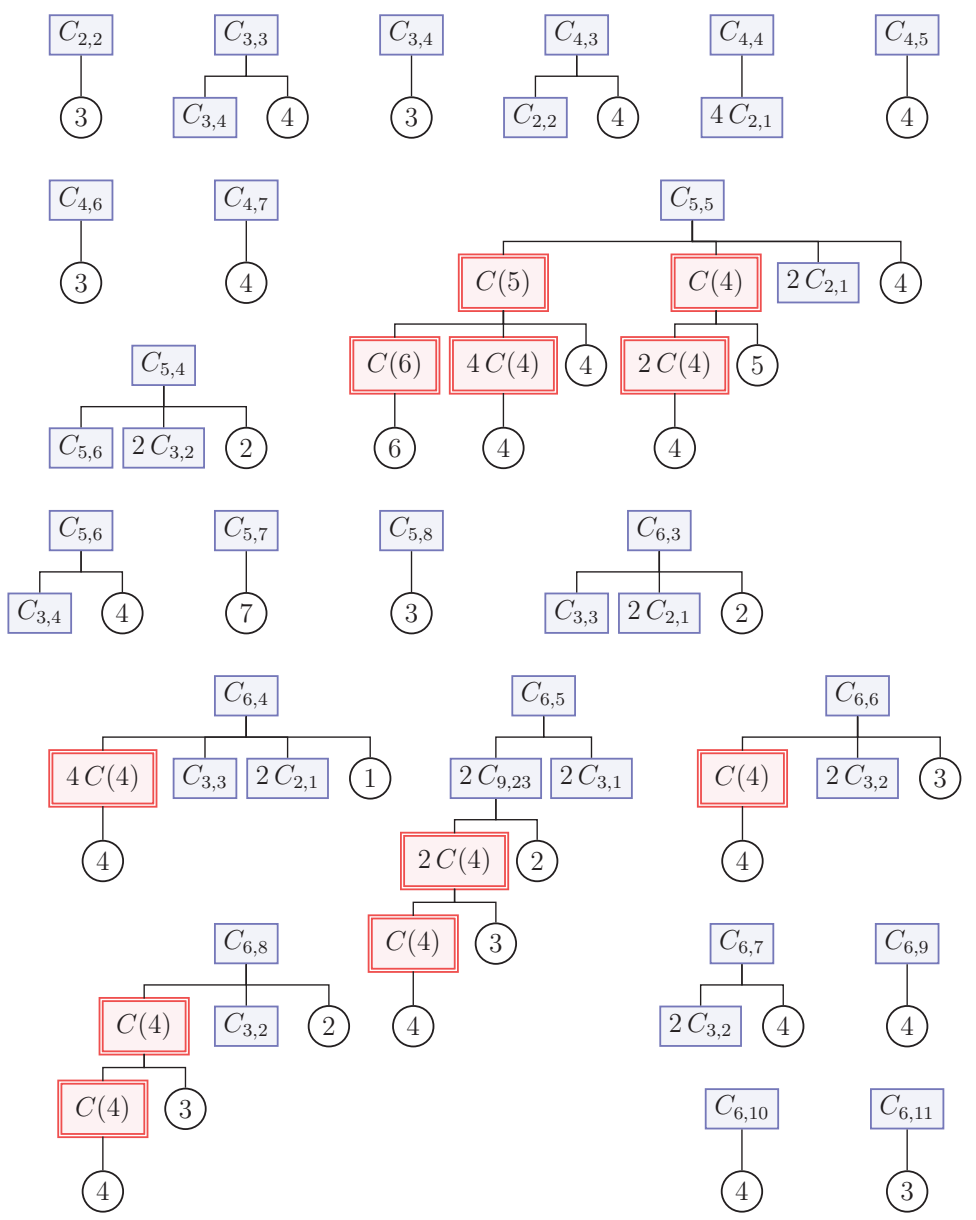

FIGURE 2. Resolution trees of irreducible simplicial cones in three dimensions.
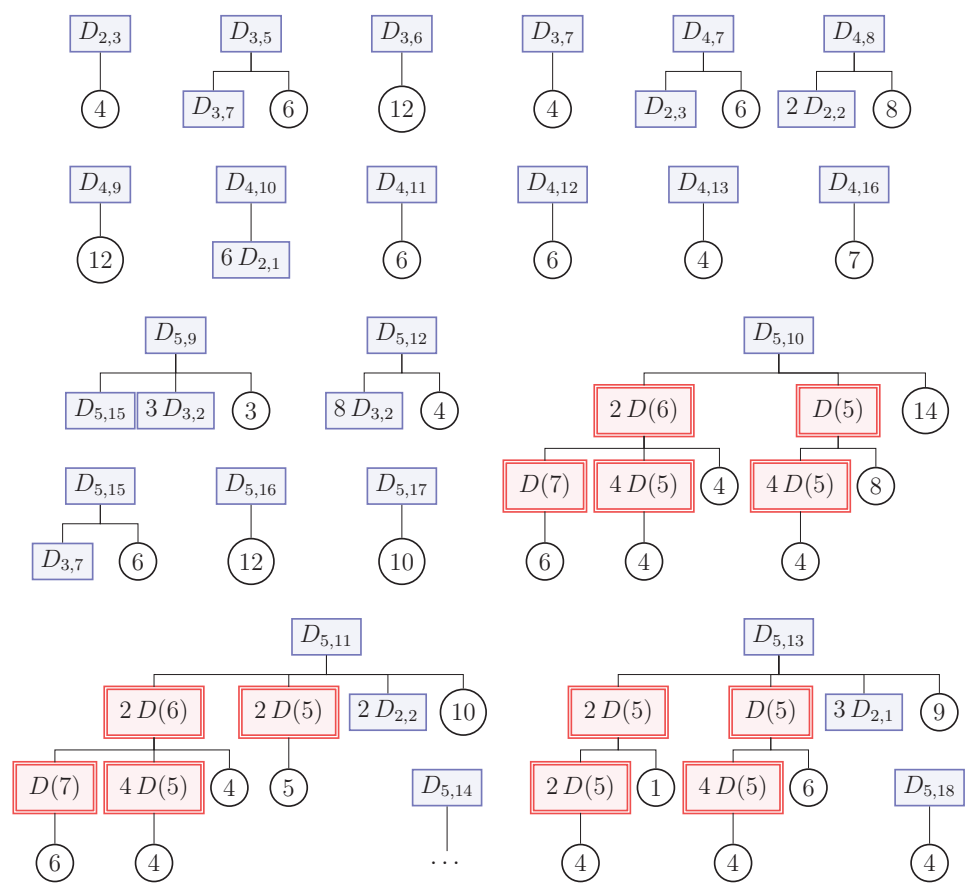

FIGURE 3. Resolution trees of irreducible simplicial cones in four dimensions. 


\begin{tabular}{|c|c|c|c|c|c|c|c|}
\hline Name & $I$ & $I^{*}$ & Pres & itati & & & Reducibility \\
\hline$D_{1,1}$ & 1 & 1 & $\left(e_{1}\right.$, & $e_{2}$, & $e_{3}$, & $\left.e_{4}\right)$ & $4 A$ \\
\hline$D_{2,1}$ & 2 & 2 & $\left(e_{1}\right.$, & $e_{2}$, & $e_{3}$ & $(0,0,1,2))$ & $B_{2,1} \oplus 2 A$ \\
\hline$D_{2,2}$ & 2 & 4 & $\left(e_{1}\right.$, & $e_{2}$ & $e_{3}$ & $(0,1,1,2))$ & $C_{2,1} \oplus A$ \\
\hline$D_{2,3}$ & 2 & 8 & $\left(e_{1}\right.$, & $e_{2}$ & $e_{3}$ & $(1,1,1,2))$ & \\
\hline$D_{3,1}$ & 3 & 3 & $\left(e_{1}\right.$, & $e_{2}$ & $e_{3}$ & $(0,0,1,3))$ & $B_{3,1} \oplus 2 A$ \\
\hline$D_{3,2}$ & 3 & 3 & $\left(e_{1}\right.$, & $e_{2}$ & $e_{3}$ & $(0,0,2,3))$ & $B_{3,2} \oplus 2 A$ \\
\hline$D_{3,3}$ & 3 & 9 & $\left(e_{1}\right.$, & $e_{2}$, & $e_{3}$ & $(0,1,1,3))$ & $C_{3,3} \oplus A$ \\
\hline$D_{3,4}$ & 3 & 9 & $\left(e_{1}\right.$, & $e_{2}$ & $e_{3}$ & $(0,2,2,3))$ & $C_{3,4} \oplus A$ \\
\hline$D_{3,5}$ & 3 & 27 & $\left(e_{1}\right.$, & $e_{2}$ & $e_{3}$ & $(1,1,1,3))$ & \\
\hline$D_{3,6}$ & 3 & 27 & $\left(e_{1}\right.$, & $e_{2}$ & $e_{3}$ & $(1,1,2,3))$ & \\
\hline$D_{3,7}$ & 3 & 27 & $\left(e_{1}\right.$, & $e_{2}$ & $e_{3}$ & $(2,2,2,3))$ & \\
\hline$D_{4,1}$ & 4 & 4 & $\left(e_{1}\right.$, & $e_{2}$ & $e_{3}$ & $(0,0,1,4))$ & $B_{4,1} \oplus 2 A$ \\
\hline$D_{4,2}$ & 4 & 4 & $\left(e_{1}\right.$, & $e_{2}$ & $e_{3}$ & $(0,0,3,4))$ & $B_{4,2} \oplus 2 A$ \\
\hline$D_{4,3}$ & 4 & 16 & $\left(e_{1}\right.$, & $e_{2}$ & $e_{3}$ & $(0,1,1,4))$ & $C_{4,3} \oplus A$ \\
\hline$D_{4,4}$ & 4 & 8 & $\left(e_{1}\right.$, & $e_{2}$ & $e_{3}$ & $(0,1,2,4))$ & $C_{4,4} \oplus A$ \\
\hline$D_{4,5}$ & 4 & 8 & $\left(e_{1}\right.$, & $e_{2}$ & $e_{3}$ & $(0,2,3,4))$ & $C_{4,5} \oplus A$ \\
\hline$D_{4,6}$ & 4 & 16 & $\left(e_{1}\right.$, & $e_{2}$ & $e_{3}$ & $(0,3,3,4))$ & $C_{4,6} \oplus A$ \\
\hline$D_{4,7}$ & 4 & 64 & $\left(e_{1}\right.$, & $e_{2}$ & $e_{3}$ & $(1,1,1,4))$ & \\
\hline$D_{4,8}$ & 4 & 32 & $\left(e_{1}\right.$, & $e_{2}$ & $e_{3}$ & $(1,1,2,4))$ & \\
\hline$D_{4,9}$ & 4 & 64 & $\left(e_{1}\right.$, & $e_{2}$ & $e_{3}$ & $(1,1,3,4))$ & \\
\hline$D_{4,10}$ & 4 & 16 & $\left(e_{1}\right.$, & $e_{2}$ & $e_{3}$ & $(1,2,2,4))$ & \\
\hline$D_{4,11}$ & 4 & 16 & $\left(e_{1}\right.$, & $e_{2}$ & $e_{3}$ & $(2,2,3,4))$ & \\
\hline$D_{4,12}$ & 4 & 32 & $\left(e_{1}\right.$, & $e_{2}$ & $e_{3}$ & $(2,3,3,4))$ & \\
\hline$D_{4,13}$ & 4 & 64 & $\left(e_{1}\right.$, & $e_{2}$ & $e_{3}$ & $(3,3,3,4))$ & \\
\hline$D_{4,14}$ & 4 & 2 & $\left(e_{1}\right.$, & $e_{2}$ & $(0,1,2,0)$ & $(0,1,0,2))$ & $C_{4,7} \oplus A$ \\
\hline$D_{4,15}$ & 4 & 4 & $\left(e_{1}\right.$, & $e_{2}$ & $(0,1,2,0)$ & $(1,0,0,2))$ & $2 B_{2,1}$ \\
\hline$D_{4,16}$ & 4 & 4 & $\left(e_{1}\right.$, & $e_{2}$ & $(0,1,2,0)$ & $(1,1,0,2))$ & \\
\hline$D_{5,1}$ & 5 & 5 & $\left(e_{1}\right.$, & $e_{2}$ & $e_{3}$ & $(0,0,1,5))$ & $B_{5,1} \oplus 2 A$ \\
\hline$D_{5,2}$ & 5 & 5 & $\left(e_{1}\right.$, & $e_{2}$ & $e_{3}$ & $(0,0,2,5))$ & $B_{5,2} \oplus 2 A$ \\
\hline$D_{5,3}$ & 5 & 5 & $\left(e_{1}\right.$, & $e_{2}$ & $e_{3}$ & $(0,0,4,5))$ & $B_{5,3} \oplus 2 A$ \\
\hline$D_{5,4}$ & 5 & 25 & $\left(e_{1}\right.$, & $e_{2}$ & $e_{3}$ & $(0,1,1,5))$ & $C_{5,4} \oplus A$ \\
\hline$D_{5,5}$ & 5 & 25 & $\left(e_{1}\right.$, & $e_{2}$ & $e_{3}$ & $(0,1,2,5))$ & $C_{5,5} \oplus A$ \\
\hline$D_{5,6}$ & 5 & 25 & $\left(e_{1}\right.$, & $e_{2}$ & $e_{3}$ & $(0,2,2,5))$ & $C_{5,6} \oplus A$ \\
\hline$D_{5,7}$ & 5 & 25 & $\left(e_{1}\right.$, & $e_{2}$ & $e_{3}$ & $(0,2,4,5))$ & $C_{5,7} \oplus A$ \\
\hline$D_{5,8}$ & 5 & 25 & $\left(e_{1}\right.$, & $e_{2}$ & $e_{3}$ & $(0,4,4,5))$ & $C_{5,8} \oplus A$ \\
\hline$D_{5,9}$ & 5 & 125 & $\left(e_{1}\right.$, & $e_{2}$ & $e_{3}$ & $(1,1,1,5))$ & \\
\hline$D_{5,10}$ & 5 & 125 & $\left(e_{1}\right.$, & $e_{2}$ & $e_{3}$ & $(1,1,2,5))$ & \\
\hline$D_{5,11}$ & 5 & 125 & $\left(e_{1}\right.$, & $e_{2}$ & $e_{3}$ & $(1,1,3,5))$ & \\
\hline$D_{5,12}$ & 5 & 125 & $\left(e_{1}\right.$, & $e_{2}$ & $e_{3}$ & $(1,1,4,5))$ & \\
\hline$D_{5,13}$ & 5 & 125 & $\left(e_{1}\right.$, & $e_{2}$ & $e_{3}$ & $(1,2,2,5))$ & \\
\hline$D_{5,14}$ & 5 & 125 & $\left(e_{1}\right.$, & $e_{2}$ & $e_{3}$ & $(1,2,3,5))$ & \\
\hline$D_{5,15}$ & 5 & 125 & $\left(e_{1}\right.$, & $e_{2}$ & $e_{3}$ & $(2,2,2,5))$ & \\
\hline$D_{5,16}$ & 5 & 125 & $\left(e_{1}\right.$, & $e_{2}$ & $e_{3}$ & $(2,2,4,5))$ & \\
\hline$D_{5,17}$ & 5 & 125 & $\left(e_{1}\right.$, & $e_{2}$ & $e_{3}$ & $(2,4,4,5))$ & \\
\hline$D_{5,18}$ & 5 & 125 & $\left(e_{1}\right.$, & $e_{2}$ & $e_{3}$ & $(4,4,4,5))$ & \\
\hline
\end{tabular}

TABLE 4. Classification of simplicial cones in four dimensions.

sprouting from simplicial cones that appear elsewhere on the page. Also, identical subtrees sprouting from the same node have been shown only once, but with the multiplicity appearing as a coefficient of the first cone on the subtree.
Furthermore, a multiple branch of the form $k C_{1,1}$ or $k D_{1,1}$ ( $k$ copies of the orthant) is denoted even more concisely by the number $k$ inside a circle. Thus, for example, the notation for $C_{5,4}$ is meant to convey that a single Nash blowup produces the five cones $C_{5,6}, C_{3,2}, C_{3,2}$, 


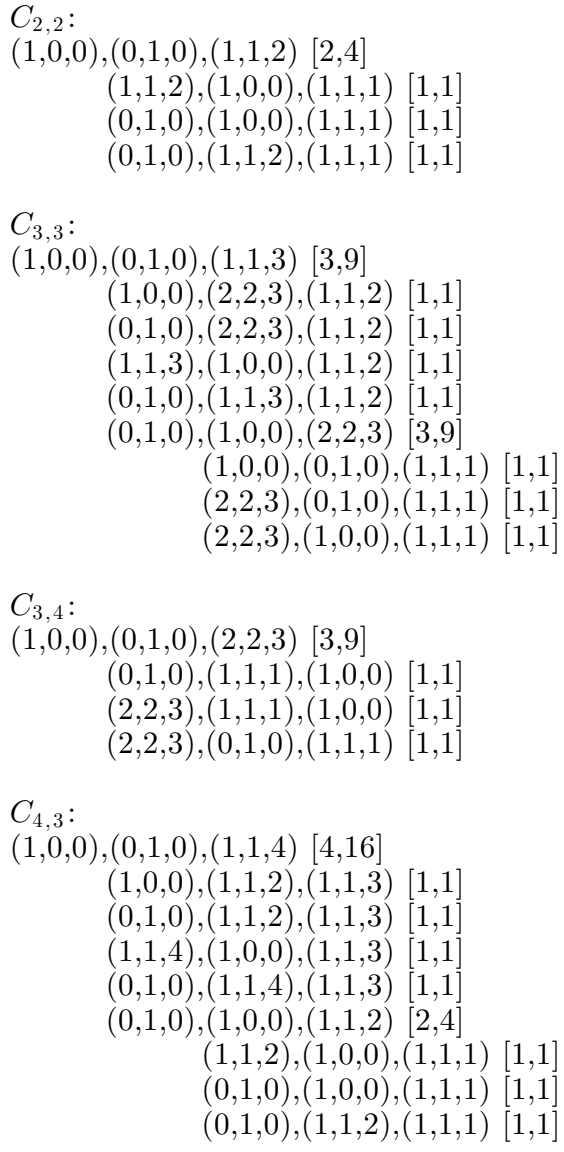

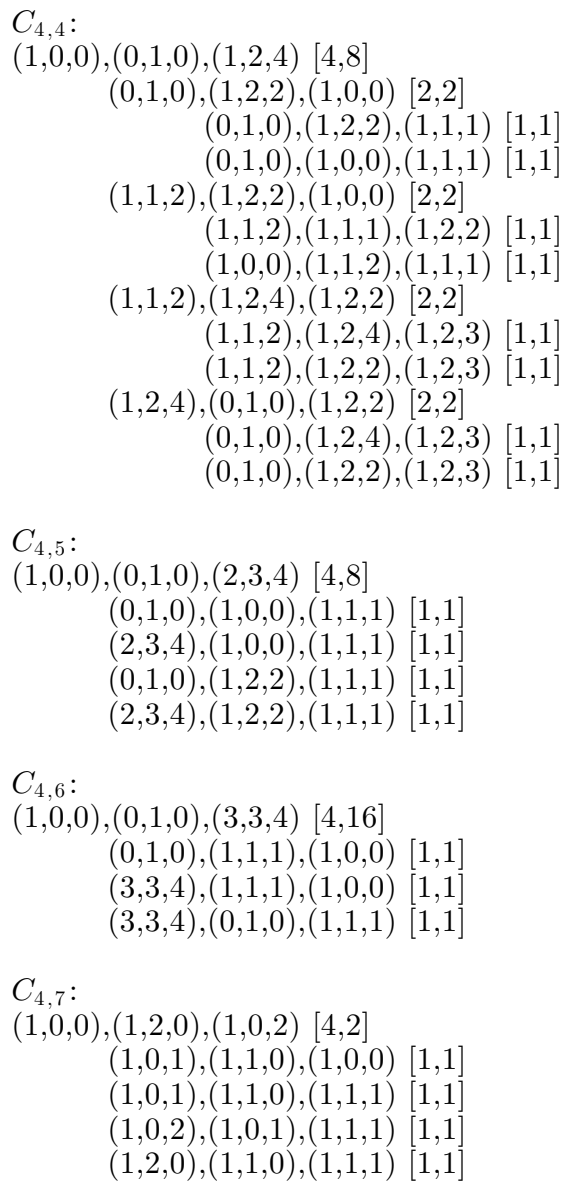

TABLE 5. Nash resolutions of irreducible simplicial cones in three dimensions.

$C_{1,1}$, and $C_{1,1}$. By definition, all leaves of a resolution tree are orthants, but this is not immediately apparent from the diagram because of the pruning convention just mentioned.

The cones appearing in double-outlined boxes are nonsimplicial cones, with the number of facets in parentheses. We did not classify these cones, so we continue their resolution trees until they reach simplicial cones encountered before. Evidently, nonsimplicial cones are ubiquitous even in the resolution of simplicial cones. (A note about the grouping of cones by multiplicity in the figures: simplicial cones have been grouped if and only if they are equivalent, whereas nonsimplicial cones are grouped if and only if they have identical resolution trees. This is a weaker condition; in some cases, such as the $4 C(4)$ in the resolution tree of $C_{5,5}$, we know that the cones in question are not equivalent.)
What patterns can be observed in the data? Most obviously, all of the thousands of cones we have studied are eventually resolved by Nash blowups. This strongly supports an affirmative answer to Question 1.2.

However, although the resolution seems always to exist, it also seems to obey neither rhyme nor reason. Almost every straightforward conjecture one might make about patterns in the Nash resolution seems to be false. We have already seen, for example, that the resolution of a simplicial cone may involve nonsimplicial cones. One might hope that the number of facets in the cone remains within some reasonable bound, but the resolutions of 4dimensional simplicial cones can require cones with as many as ten facets, with no end in sight.

The behavior of the indices $I$ and $I^{*}$ is equally perplexing. A 2-dimensional cone $\mathbb{Q}_{+}\langle(1,0),(p, q)\rangle$ with $p$ coprime to $q$ has $I(C)=I^{*}(C)=q$. As we saw 
$D_{2,3}$ :

$(1,0,0,0),(0,1,0,0),(0,0,1,0),(1,1,1,2)[2,8]$

$(0,0,1,0),(1,1,1,2),(1,0,0,0),(1,1,1,1)[1,1$ $(0,1,0,0),(1,1,1,2),(1,0,0,0),(1,1,1,1)[1,1$ $(0,1,0,0),(0,0,1,0),(1,0,0,0),(1,1,1,1)[1,1]$ $(0,1,0,0),(0,0,1,0),(1,1,1,2),(1,1,1,1)[1,1]$

$D_{3,5}$ :

$(1,0,0,0),(0,1,0,0),(0,0,1,0),(1,1,1,3)[3,27]$ $(0,0,1,0),(1,0,0,0),(2,2,2,3),(1,1,1,2)[1,1$ $(0,1,0,0),(1,0,0,0),(2,2,2,3),(1,1,1,2)[1,1]$ $(0,1,0,0),(0,0,1,0),(2,2,2,3),(1,1,1,2)[1,1]$ $(0,0,1,0),(1,1,1,3),(1,0,0,0),(1,1,1,2)[1,1$ $(0,1,0,0),(1,1,1,3),(1,0,0,0),(1,1,1,2)[1,1$ $(0,1,0,0),(0,0,1,0),(1,1,1,3),(1,1,1,2)[1,1]$ $(0,1,0,0),(0,0,1,0),(1,0,0,0),(2,2,2,3)[3,27]$ $(2,2,2,3),(1,0,0,0),(0,0,1,0),(1,1,1,1)[1,1]$ $(0,1,0,0),(1,0,0,0),(0,0,1,0),(1,1,1,1)[1,1$ $(0,1,0,0),(2,2,2,3),(0,0,1,0),(1,1,1,1)[1,1]$ $(0,1,0,0),(2,2,2,3),(1,0,0,0),(1,1,1,1)[1,1]$

$D_{3,6}$ :

$(1,0,0,0),(0,1,0,0),(0,0,1,0),(1,1,2,3)[3,27]$ $(0,0,1,0),(1,0,0,0),(2,2,3,3),(1,1,1,1)[1,1]$ $(1,1,2,3),(1,0,0,0),(2,2,3,3),(1,1,1,1)[1,1$ $(0,1,0,0),(1,1,2,3),(2,2,3,3),(1,1,1,1)[1,1$ $(0,1,0,0),(1,1,2,3),(1,0,0,0),(1,1,1,1)[1,1]$ $(0,1,0,0),(0,0,1,0),(2,2,3,3),(1,1,1,1)[1,1$ $(0,1,0,0),(0,0,1,0),(1,0,0,0),(1,1,1,1)[1,1$ $(0,0,1,0),(1,1,2,2),(1,0,0,0),(2,2,3,3)[1,1$ $(1,1,2,3),(1,1,2,2),(1,0,0,0),(2,2,3,3)[1,1]$ $(0,1,0,0),(1,1,2,3),(1,1,2,2),(2,2,3,3)[1,1$ $(0,1,0,0),(0,0,1,0),(1,1,2,2),(2,2,3,3)[1,1$ $(1,1,2,3),(0,0,1,0),(1,1,2,2),(1,0,0,0)[1,1]$ $(0,1,0,0),(1,1,2,3),(0,0,1,0),(1,1,2,2)[1,1]$

$D_{3,7}$ :

$(1,0,0,0),(0,1,0,0),(0,0,1,0),(2,2,2,3)[3,27]$

$(2,2,2,3),(0,0,1,0),(1,1,1,1),(1,0,0,0)[1,1$ $(0,1,0,0),(0,0,1,0),(1,1,1,1),(1,0,0,0)[1,1$ $(0,1,0,0),(2,2,2,3),(1,1,1,1),(1,0,0,0)[1,1$ $(0,1,0,0),(2,2,2,3),(0,0,1,0),(1,1,1,1)[1,1]$

$D_{4,10}$ :

$(1,0,0,0),(0,1,0,0),(0,0,1,0),(1,2,2,4)[4,16]$

$(1,1,1,2),(0,1,0,0),(1,2,2,2),(1,0,0,0)[2,2]$ $(0,1,0,0),(1,1,1,2),(1,1,1,1),(1,2,2,2)[1,1]$ $(1,0,0,0),(0,1,0,0),(1,1,1,2),(1,1,1,1)[1,1]$ $(0,0,1,0),(0,1,0,0),(1,2,2,2),(1,0,0,0)[2,2]$ $(0,1,0,0),(0,0,1,0),(1,2,2,2),(1,1,1,1)[1,1]$ $(0,1,0,0),(0,0,1,0),(1,0,0,0),(1,1,1,1)[1,1]$ $(0,0,1,0),(1,1,1,2),(1,2,2,2),(1,0,0,0)[2,2]$ $(1,1,1,2),(0,0,1,0),(1,1,1,1),(1,2,2,2)[1,1]$ $(1,0,0,0),(1,1,1,2),(0,0,1,0),(1,1,1,1)[1,1]$

$(1,1,1,2),(1,2,2,4),(0,1,0,0),(1,2,2,2)[2,2]$ $(0,1,0,0),(1,1,1,2),(1,2,2,4),(1,2,2,3)[1,1]$ $(0,1,0,0),(1,1,1,2),(1,2,2,2),(1,2,2,3)[1,1]$

$(0,0,1,0),(1,2,2,4),(0,1,0,0),(1,2,2,2)[2,2]$ $(0,1,0,0),(0,0,1,0),(1,2,2,4),(1,2,2,3)[1,1]$ $(0,1,0,0),(0,0,1,0),(1,2,2,2),(1,2,2,3)[1,1]$

$(0,0,1,0),(1,1,1,2),(1,2,2,4),(1,2,2,2)[2,2]$ $(0,0,1,0),(1,1,1,2),(1,2,2,4),(1,2,2,3)[1,1$ $(0,0,1,0),(1,1,1,2),(1,2,2,2),(1,2,2,3)[1,1]$
$D_{4,7}$ :

$(1,0,0,0),(0,1,0,0),(0,0,1,0),(1,1,1,4)[4,64]$

$(0,0,1,0),(1,0,0,0),(1,1,1,2),(1,1,1,3)[1,1$ $(0,1,0,0),(1,0,0,0),(1,1,1,2),(1,1,1,3)[1,1]$ $(0,1,0,0),(0,0,1,0),(1,1,1,2),(1,1,1,3)[1,1]$ $(0,0,1,0),(1,1,1,4),(1,0,0,0),(1,1,1,3)[1,1$ $(0,1,0,0),(1,1,1,4),(1,0,0,0),(1,1,1,3)[1,1]$ $(0,1,0,0),(0,0,1,0),(1,1,1,4),(1,1,1,3)[1,1]$ $(0,1,0,0),(0,0,1,0),(1,0,0,0),(1,1,1,2)[2,8]$ $(0,0,1,0),(1,1,1,2),(1,0,0,0),(1,1,1,1)[1,1]$ $(0,1,0,0),(1,1,1,2),(1,0,0,0),(1,1,1,1)[1,1$ $(0,1,0,0),(0,0,1,0),(1,0,0,0),(1,1,1,1)[1,1]$ $(0,1,0,0),(0,0,1,0),(1,1,1,2),(1,1,1,1)[1,1]$

$D_{4,8}$ :

$(1,0,0,0),(0,1,0,0),(0,0,1,0),(1,1,2,4)[4,32]$ $(1,0,0,0),(1,1,1,2),(1,1,2,2),(1,1,2,3)[1,1]$ $(0,1,0,0),(1,1,1,2),(1,1,2,2),(1,1,2,3)[1,1]$ $(0,0,1,0),(1,0,0,0),(1,1,2,2),(1,1,2,3)[1,1]$ $(0,1,0,0),(0,0,1,0),(1,1,2,2),(1,1,2,3)[1,1]$ $(1,1,2,4),(0,0,1,0),(1,0,0,0),(1,1,2,3)[1,1]$ $(0,1,0,0),(1,1,2,4),(0,0,1,0),(1,1,2,3)[1,1]$ $(0,1,0,0),(0,0,1,0),(1,0,0,0),(1,1,2,2)[2,4]$ $(0,0,1,0),(1,1,2,2),(1,0,0,0),(1,1,1,1)[1,1]$ $(0,1,0,0),(0,0,1,0),(1,0,0,0),(1,1,1,1)[1,1$ $(0,1,0,0),(0,0,1,0),(1,1,2,2),(1,1,1,1)[1,1]$ $(1,1,2,4),(1,0,0,0),(1,1,1,2),(1,1,2,3)[1,1]$ $(0,1,0,0),(1,1,2,4),(1,1,1,2),(1,1,2,3)[1,1]$ $(0,1,0,0),(1,0,0,0),(1,1,1,2),(1,1,2,2)[2,4]$ $(0,1,0,0),(1,1,1,2),(1,1,2,2),(1,1,1,1)[1,1]$ $(1,0,0,0),(1,1,1,2),(1,1,2,2),(1,1,1,1)[1,1$ $(1,0,0,0),(0,1,0,0),(1,1,1,2),(1,1,1,1)[1,1]$

$D_{4,9}:$

$(1,0,0,0),(0,1,0,0),(0,0,1,0),(1,1,3,4)[4,64]$ $(0,0,1,0),(1,0,0,0),(1,1,2,2),(1,1,3,3)[1,1]$ $(0,1,0,0),(0,0,1,0),(1,1,2,2),(1,1,3,3)[1,1]$ $(1,1,3,4),(1,0,0,0),(1,1,2,2),(1,1,3,3)[1,1]$ $(0,1,0,0),(1,1,3,4),(1,1,2,2),(1,1,3,3)[1,1]$ $(1,1,3,4),(0,0,1,0),(1,0,0,0),(1,1,3,3)[1,1]$ $(0,1,0,0),(1,1,3,4),(0,0,1,0),(1,1,3,3)[1,1]$ $(0,0,1,0),(1,0,0,0),(1,1,1,1),(1,1,2,2)[1,1$ $(0,1,0,0),(0,0,1,0),(1,1,1,1),(1,1,2,2)[1,1$ $(1,1,3,4),(1,0,0,0),(1,1,1,1),(1,1,2,2)[1,1]$ $(0,1,0,0),(1,1,3,4),(1,1,1,1),(1,1,2,2)[1,1]$ $(0,1,0,0),(1,1,3,4),(1,0,0,0),(1,1,1,1)[1,1]$

$D_{4,11}$ $(0,1,0,0),(0,0,1,0),(1,0,0,0),(1,1,1,1)[1,1]$

$(1,0,0,0),(0,1,0,0),(0,0,1,0),(2,2,3,4)[4,16]$ $(1,1,1,1),(0,0,1,0),(1,1,2,2),(1,0,0,0)[1,1]$ $(2,2,3,4),(1,1,1,1),(1,1,2,2),(1,0,0,0)[1,1]$ $(0,1,0,0),(1,1,1,1),(0,0,1,0),(1,0,0,0)[1,1]$ $(2,2,3,4),(0,1,0,0),(1,1,1,1),(1,0,0,0)[1,1]$ $(0,1,0,0),(1,1,1,1),(0,0,1,0),(1,1,2,2)[1,1]$ $(2,2,3,4),(0,1,0,0),(1,1,1,1),(1,1,2,2)[1,1]$

$D_{4,12}$ :

$(1,0,0,0),(0,1,0,0),(0,0,1,0),(2,3,3,4)[4,32]$ $(0,1,0,0),(0,0,1,0),(1,2,2,2),(1,1,1,1)[1,1]$ $(2,3,3,4),(0,0,1,0),(1,2,2,2),(1,1,1,1)[1,1]$ $(1,0,0,0),(2,3,3,4),(0,0,1,0),(1,1,1,1)[1,1]$ $(2,3,3,4),(0,1,0,0),(1,2,2,2),(1,1,1,1)[1,1]$ $(1,0,0,0),(2,3,3,4),(0,1,0,0),(1,1,1,1)[1,1]$ $(1,0,0,0),(0,1,0,0),(0,0,1,0),(1,1,1,1)[1,1]$

$D_{4,13}$ :

$(1,0,0,0),(0,1,0,0),(0,0,1,0),(3,3,3,4)[4,64]$ $(0,1,0,0),(0,0,1,0),(1,1,1,1),(1,0,0,0)[1,1]$ $(3,3,3,4),(0,0,1,0),(1,1,1,1),(1,0,0,0)[1,1]$ $(3,3,3,4),(0,1,0,0),(1,1,1,1),(1,0,0,0)[1,1]$ $(3,3,3,4),(0,1,0,0),(0,0,1,0),(1,1,1,1)[1,1]$

TABLE 6. Nash resolutions of irreducible simplicial cones in four dimensions. 
in Proposition 4.13, this is nonincreasing under Nash blowup (indeed, decreasing except for $p$ odd and $q=p-$ 1). But $I$ and $I^{*}$ can increase under Nash blowups, even in dimension 3 and even when the cones involved are simplicial. For example, $C_{6,5}=\mathbb{Q}_{+}\langle(1,0,0),(0,1,0),(1,3,6)\rangle$ with $I=6$ gives rise, after a single Nash blowup, to $\mathbb{Q}_{+}\langle(1,3,6),(1,3,3),(2,3,6)\rangle \cong C_{9,23}$ with $I=9$. A glimmer of hope is offered by $I^{*}$. For among the thousands of cones we have examined, there appears not one example of a simplicial cone giving rise, after a single Nash blowup, to another simplicial cone with greater $I^{*}$. However, there are rare cases in which, after two Nash blowups, one obtains a simplicial cone with greater $I^{*}$. For example, $C_{9,22}=\mathbb{Q}_{+}\langle(1,0,0),(1,3,0),(1,0,3)\rangle$ with $I^{*}=3$ gives rise, after two Nash blowups, to $\mathbb{Q}_{+}\langle(1,1,0),(1,0,1),(4,3,3)\rangle$ and two other cones all with $I^{*}=4$. Moreover, there are many cases in which $I^{*}$ increases when one of the cones is not simplicial. This can be seen, for example, in the resolution tree of $C_{7,6}$, where $\mathbb{Q}_{+}\langle(1,0,0),(0,1,0),(2,4,7),(1,1,2)\rangle$ with $I^{*}=1$ gives rise, after a single Nash blowup, to $\mathbb{Q}_{+}\langle(1,0,0),(0,1,0),(1,2,2)\rangle \cong C_{2,1}$ with $I^{*}=2$.

The question is reminiscent of other famous iterative problems such as the notorious Collatz conjecture [Lagarias 85], but in some ways it is even worse behaved. A striking empirical feature is the existence of simplicial cones whose Nash resolution is vastly larger than those of other simplicial cones with the same index. In dimension 4 , for example, the seemingly innocent $D_{5,14}=$ $\mathbb{Q}_{+}\left\langle e_{1}, e_{2}, e_{3},(1,2,3,5)\right\rangle$, with $I=5$, has a resolution tree with depth 8 and 14,253 cones, while no other simplicial cone with $I=5$ needs more than depth 3 and 108 cones. Likewise, $D_{7,24}=\mathbb{Q}_{+}\left\langle e_{1}, e_{2}, e_{3},(1,2,5,7)\right\rangle$, with $I=7$, has a resolution tree with depth 11 and 35,299 cones, while no other simplicial cone with $I=7$ needs more than depth 7 and 5061 cones, and only one other needs more than depth 5 and 804 cones.

In conclusion, Question 1.2 remains wide open, but we have amassed considerable empirical evidence supporting an affirmative answer. In light of the 2-dimensional case, one might hope for a proof involving some kind of higherdimensional analogue of continued fractions.

\section{ACKNOWLEDGMENTS}

The very helpful advice provided by Kevin Purbhoo is gratefully acknowledged. We also thank Jeffrey Lagarias for an inspiring conversation, Dave Bayer for recommending the use of 4ti2, and Sam Payne and Greg Smith for informing us of their parallel work on the subject. Nicholas Proudfoot's work was supported by NSF grant DMS-0738335; Michael Thaddeus's work was supported by NSF grants DMS-0401128 and DMS-0700419.

\section{REFERENCES}

[Ewald 96] G. Ewald. Combinatorial Convexity and Algebraic Geometry, Grad. Texts in Math. 168. New York: Springer, 1996.

[Fulton 93] W. Fulton. Introduction to Toric Varieties, Ann. of Math. Stud. 131. Princeton: Princeton Univ. Press, 1993.

[Gonzalez-Sprinberg 77a] G. Gonzalez-Sprinberg. "Transformé de Nash et éventail de dimension 2." C. R. Acad. Sci. Paris Sér. A-B 284 (1977), A69-A71.

[Gonzalez-Sprinberg 77b] G. Gonzalez-Sprinberg. Éventails en dimension deux et transformé de Nash. Paris: Publications de l'École Normale Supérieure, 1977. (Available online at http://www-fourier.ujf-grenoble.fr/ gonsprin/ publications-ggs.html).

[Gonzalez-Sprinberg 87] G. Gonzalez-Sprinberg. "Désingularisation des surfaces par des modifications de Nash normalisées (d'après M. Spivakovsky), Sém. Bourbaki 1985/86." Astérisque 145/146 (1987) 187-207.

[Hartshorne 77] R. Hartshorne. Algebraic Geometry, Grad. Texts in Math. 52. New York: Springer, 1977.

[Hironaka 83] H. Hironaka. "On Nash Blowing-Up." In Arithmetic and Geometry, vol. II, edited by M. Artin and J. Tate, Progr. Math. 36, pp. 103-111. Boston: Birkhäuser, 1983.

[Lagarias 85] J. C. Lagarias. "The $3 x+1$ Problem and Its Generalizations." Amer. Math. Monthly 92 (1985), 3-23.

[Lazarsfeld 04] R. Lazarsfeld. Positivity in Algebraic Geometry I, Ergeb. Math. 48. New York: Springer, 2004.

[Miller and Sturmfels 05] E. Miller and B. Sturmfels. Combinatorial Commutative Algebra, Grad. Texts in Math. 227. New York: Springer, 2005.

[Nobile 75] A. Nobile. "Some Properties of the Nash BlowingUp." Pac. J. Math. 60 (1975), 297-305.

[Schrijver 86] A. Schrijver. Theory of Linear and Integer Programming. New York: Wiley, 1986.

[Spivakovsky 90] M. Spivakovsky. "Sandwiched Singularities and Desingularization of Surfaces by Normalized Nash Transformations." Ann. of Math. (2) 131 (1990), 411491. 
[Thaddeus 94] M. Thaddeus. "Toric Quotients and Flips." In Topology, Geometry and Field Theory, edited by K.
Fukaya, M. Furuta, T. Kohno, and D. Kotschick, pp. 193213. Singapore: World Scientific, 1994.

Atanas Atanasov, Department of Mathematics, Columbia University, 2990 Broadway, New York, NY 10027, USA (nasko@math.harvard.edu)

Christopher Lopez, Department of Mathematics, Columbia University, 2990 Broadway, New York, NY 10027, USA (clopez04971@gmail.com)

Alexander Perry, Department of Mathematics, Columbia University, 2990 Broadway, New York, NY 10027, USA (arp2125@columbia.edu)

Nicholas Proudfoot, Department of Mathematics, University of Oregon, 1222 University of Oregon, Eugene, OR 97403, USA (njp@uoregon.edu)

Michael Thaddeus, Department of Mathematics, Columbia University, 2990 Broadway, New York, NY 10027, USA (thaddeus@math.columbia.edu)

Received October 26, 2009; accepted May 13, 2010. 